\title{
Converged Statistics for Time-Resolved Measurements in Low- Speed Axial Fans using High-Frequency Response Probes
}

\author{
Fernández Oro, J.M. ${ }^{(1)}$; Argüelles Díaz, K.M.; \\ Rodríguez Lastra, M.; Galdo Vega, M.; Pereiras García, B. \\ Universidad de Oviedo, Área de Mecánica de Fluidos. \\ Campus de Viesques, 33271, Gijón (Asturias), Spain. \\ (1) jesusfo@uniovi.es
}

\begin{abstract}
Fast-response probes in multistage turbomachinery are typically used to measure unsteady flows and turbulence in a number of traverse locations throughout the machine (rotor-stator interregions, inlet and outlet sections, tip clearance gaps...). When used intensively, they provide complete information of time-resolved flow structures, including wake patterns, wake mixing, wake-wake and rotor-wake interactions or turbulence transport in 2D planes and even 3D pictures if the raw signals are post-processed accurately.

The segregation between deterministic, unsteady features and turbulent scales is essential to understand the unsteady mechanisms responsible for the energy transfer and requires an accurate selection of the sampling frequencies and the total length of the measured traces to assure a valid statistical reduction. Similar considerations must be made if they are filtered in a frequency basis (for example, filtering low-scale turbulence or extracting only BPF components), employing welldesigned periodograms or power spectra with minimum scatter and large periods of time integration.

This work presents the effect of number of periods (ensembles), resolution in which the averaged periods are reconstructed and turbulence intensity on the experimental accuracy of ensemble-averaged measurements in low-speed axial fans using fast-response probes. In particular, the statistical analysis is established in terms of convergence (residuals) between timeresolved traces retrieved using different sampling frequencies and number of total samples. The possible effects of three-dimensionality, the measured regions (hub, tip, midspan) or the sensibility to turbulence levels is also explored.

A technique to quantify the convergence of the phase-locked averaging (PLA) processes is applied to a low-speed axial fan, with twin configurations of rotor-stator and stator-rotor arrangements. As a starting point, a concise survey of usual practices employed by other authors in the literature for axial fans and compressors is firstly reviewed, in order to identify fundamental parameters and values typically adopted to guarantee convergence. Finally, typical requirements are given as a function of the variable analyzed, the wake pattern to be described or the global disorder of the flow structures inside axial flow fans.
\end{abstract}

Keywords: statistical convergence, phase-locked averaging, hot-wire anemometry, axial fan, timeresolved measurements, unsteady flow, rotor-stator, high-frequency response probes. 


\section{1.- INTRODUCTION}

In the last two decades, the phase-locked averaging technique has been widely used in combination with hot-wire anemometry and fast-response pressure probes to study the flow inside axial turbomachinery. To segregate between deterministic and non-deterministic scales, it is necessary to pay particular attention to the number of samples adopted per blade passing period, as well as the total number of samples considered for an accurate statistical reduction of the velocity traces.

The number of samples for each blade passing period gives a good idea of the circumferential precision of the measurements. This value is related to both acquiring (sampling frequency) and blade passing frequencies (BPF), and also to the number of rotor blades. An accurate value is essential to obtain a precise description of the gradients associated to the wake shear layers when measuring with stationary probes. Depending on the subject of the study or the phenomena under study, this value may require a higher number (i.e., high-complex structures developed under near-stall conditions) or a lower number of samples (primary flow at design conditions). However, the own intrinsic limitations of the dynamic response of the instrumentation plays here a major role. Effectively, the resolution of the period strongly depends on the dynamic response of the measuring technique, which cannot be exceeded. This cannot be exclusively determined a priori on the only basis of post-processing considerations, and as a consequence, preliminary analysis in the frequency domain is mandatory to assure that the probes performance is consistent with the unsteadiness to be measured. Hence, it is necessary to be sure that frequency response of the anemometer is high enough to sample at the required rates.

On the other hand, the total number of samples depends only on the length of the acquiring time. That is, more samples are stored if the acquiring time is increased. Unfortunately, this time cannot be indefinitely extended because the store space or the duration of the tests is limited. Although this is rarely a problem in the case of low-speed turbomachinery applications with blade passing frequencies at least in the order of one $\mathrm{kHz}$ (where a few hundred of ensembles are recorded in one second of acquisition), it could turn to be a limitation in terms of total amount of data recorded in the case of intensive measurements performed in multiple locations (i.e, traverse measuring planes). Furthermore, it can be a major concern in the case of short-duration facilities in which the measurement window is a few tenths of a second, or in the case of slow-rotating machines, like wind turbines. In such applications, the minimum number of samples that must be adopted to provide a converged description of the time-resolved flow structures can be valuable information.

Table 1 shows a concise review of typical parameters employed for data-acquisition techniques used in multistage environments of axial fans and compressors. Concerning the number of samples for each blade passing period, the tangential discretization is shown to be in the order of one point per degree. In particular, most of these studies adopted a number of points ranging from 50 to 100 samples per blade passing period. Maximum values, like Huyer and Snarski [1], employed 167 points corresponding to 0.36 points per degree for a six-bladed diagonal flow fan. Similarly, Kergourlay et al. [2] used 125 points in a single low-speed axial fan with a 6-blade rotor resulting in 2.08 points per degree. However, it is not unusual to consider even a lower number of samples: Read and Elder [3] employed 43 samples (8.95 points per degree); Mailach and Vogeler [4], 49 samples (8.57); Velarde-Suárez el al. [5], 32 samples (0.71) and Prato et al. [6], 20 samples (4.11); or more recently, Lepicovsky [7], introducing 16 samples to obtain 625 points for a complete revolution (1.73). 
Complementarily, regarding the total number of samples to be used in the ensemble-averaging process, typical values range from 100 to 300 in the literature [8]. This disparity depends on the levels of turbulence, secondary flows and the global disturbance of the flow field. Note that maximum values reported in table 1, like those by Henderson et al. [9] with 3072 ensembles, or Senkter and Reiss [10] with 3800, are related to turbulence measurements. Conversely, several authors have introduced a limited number of samples, in accordance to Brunn guidelines, without severe lack of fidelity. For example, Lakshminarayana [11-12] employed 80 ensembles; Camp and Shin [13] adopted 45 samples for a rotor periodicity; Huyer and Snarski stored 49 blade passages and Lepicovsky sampled a full-annulus with 65 samples in a 4-stage low-speed axial compressor.

Recently, the increasing use of experimental investigations based on optical measurement techniques has renewed the interest for converged statistics in unsteady flows. In particular, early contributions can be found in Uzol and Camci [14], Perrin et al. [15] or Ullum et al. [16] in the case of canonical flows like cylinder wakes or squared fences. In the case of pitching or rotating flows, Wernert and Favier [17] and Cavazzini et. al [18] had presented valuable procedures for validating turbulent unsteady flows in turbomachinery environments. In a similar fashion, taking advantage of the mimic between fully unsteady codes and time-resolved measurements, Clark and Glover [19] had also presented a quantitative method based on fuzzy logic to assess the level of convergence of a periodic-unsteady simulation for axial turbines. Following the same basic concepts, this investigation presents a rational technique to quantify the convergence of timeresolved measurements in multistage environments of axial flow fans and compressors using intrusive probes. Basic parameters in the data acquisition of the phase-locked averaging technique, related to the number of ensembles and the number of samples per blade passing period, are used to define indicators of convergence in terms of normalized residuals and discretization error. The methodology is afterwards applied within an experimental database for time-resolved measurements of unsteady flow and turbulence in a single-stage, low-speed axial fan with rotor-stator and stator-rotor configurations. Different convergence criteria are defined as a function of the variable of interest (velocity or turbulence intensity) and the influence of probe locations respect to boundary layers affection or wakes shear layers is also explored. The obtained results and the basics of the rational technique presented here can be of particular interest in the case of short-duration facilities or slow-rotating machines where acquiring times and sampling frequencies can be an important restriction to obtain reliable measurements.

Table 1. Typical values employed in the literature for accurate measurements in low-speed axial compressors and fans (B: Number of rotor blades; BPF: Blade passing frequency; $f_{s}$ : Sampling frequency; M: Number of Ensemble averages; N: Number of Samples per blade event).

\begin{tabular}{|c|c|c|c|c|c|c|c|c|c|c|}
\hline Author (s) & Year & Sensor type & $\begin{array}{l}\text { Subject of } \\
\text { study }\end{array}$ & $\begin{array}{l}\text { Type of } \\
\text { machine }\end{array}$ & RPM & B & $\begin{array}{l}\text { BPF } \\
\text { (Hz) }\end{array}$ & $\begin{array}{c}\text { fs } \\
(\mathrm{kHz})\end{array}$ & $\mathbf{M}$ & $\mathbf{N}$ \\
\hline $\begin{array}{l}\text { Lakshminarayana } \\
\text { and Poncet [11] }\end{array}$ & 1974 & $\begin{array}{l}X \& \text { single } \\
\text { hot-wire } \\
\text { sensor }\end{array}$ & Rotor wakes & $\begin{array}{l}\text { Axial flow } \\
\text { inducer }\end{array}$ & 450 & 3 & 30 & 1.5 & 80 & 50 \\
\hline $\begin{array}{l}\text { Lakshminarayana } \\
\text { [12] }\end{array}$ & 1981 & $\begin{array}{l}\text { Triple- } \\
\text { sensor hot- } \\
\text { wire }\end{array}$ & Passage flow & $\begin{array}{l}\text { Axial flow } \\
\text { inducer }\end{array}$ & 450 & 4 & 22.5 & 1.1 & 80 & 50 \\
\hline $\begin{array}{l}\text { Inoue and } \\
\text { Kuroumaru [32] }\end{array}$ & 1989 & $\begin{array}{l}\text { Slanted } \\
\text { single hot- } \\
\text { wire }\end{array}$ & $\begin{array}{l}\text { Tip vortex, } \\
\text { clearance } \\
\text { flow }\end{array}$ & $\begin{array}{l}\text { Single axial } \\
\text { compressor }\end{array}$ & 1300 & 16 & 346.7 & 17.3 & 512 & 50 \\
\hline Dong and Cumptsy & 1990 & Hot-wire & Boundary & Compressor & - & - & 17 & 5.4 & 100 & 320 \\
\hline
\end{tabular}




\begin{tabular}{|c|c|c|c|c|c|c|c|c|c|c|}
\hline [33] & & $\begin{array}{l}\text { (single } \\
\text { sensor) }\end{array}$ & layers & $\begin{array}{l}\text { cascade with } \\
\text { upstream } \\
\text { rods }\end{array}$ & & & & & & \\
\hline Goto [21] & 1992 & $\begin{array}{l}\text { Slanted } \\
\text { single hot- } \\
\text { wire }\end{array}$ & $\begin{array}{l}\text { Tip vortex, } \\
\text { 3D flow } \\
\text { structures }\end{array}$ & $\begin{array}{l}\text { Single axial } \\
\text { compressor }\end{array}$ & 500 & 51 & 425 & 23.8 & 816 & 56 \\
\hline Camp and Shin [13] & 1995 & $\begin{array}{l}\text { Hot-wire } \\
\text { (single } \\
\text { sensor) }\end{array}$ & $\begin{array}{l}\text { Turbulence } \\
\text { intensity, } \\
\text { scales }\end{array}$ & $\begin{array}{l}\text { 4-stage axial } \\
\text { compressor }\end{array}$ & 840 & 79 & 1109 & 100.9 & 45 & 91 \\
\hline $\begin{array}{l}\text { Witkowski et al. } \\
\text { [34] }\end{array}$ & 1996 & $\begin{array}{l}\text { Hot-film } \\
\text { (triple-split) }\end{array}$ & $\begin{array}{l}\text { 3D wake } \\
\text { decay }\end{array}$ & $\begin{array}{l}\text { Axial } \\
\text { compressor }\end{array}$ & 800 & 16 & 213.3 & 10.7 & 1000 & 50 \\
\hline Halstead et al. [35] & 1997 & $\begin{array}{l}\text { Hot-wire, } \\
\text { hot-film }\end{array}$ & $\begin{array}{l}\text { Unsteady } \\
\text { Boundary } \\
\text { layers }\end{array}$ & $\begin{array}{l}\text { 4-stage axial } \\
\text { compressor }\end{array}$ & 840 & 54 & 756 & 100 & $\begin{array}{c}200- \\
400\end{array}$ & $\begin{array}{l}100- \\
500\end{array}$ \\
\hline $\begin{array}{l}\text { Sentker and Reiss } \\
{[10]}\end{array}$ & 1998 & $\begin{array}{l}\text { Split hot- } \\
\text { film }\end{array}$ & $\begin{array}{l}\text { Turbulence, } \\
\text { unsteadines } \\
\text { S }\end{array}$ & $\begin{array}{l}\text { 2-stage axial } \\
\text { compressor }\end{array}$ & 3000 & 30 & 1500 & 200 & 3800 & 138 \\
\hline Furukawa [36] & 1998 & $\begin{array}{l}\text { Hot-wire } \\
\text { (slanted } \\
\text { single) }\end{array}$ & $\begin{array}{l}\text { Tip } \\
\text { clearance } \\
\text { flow field }\end{array}$ & Axial rotor & 1300 & 12 & 260 & 26 & 150 & 100 \\
\hline $\begin{array}{l}\text { Quinlan and Bent } \\
\text { [37] }\end{array}$ & 1998 & $\begin{array}{l}\text { Hot- } \\
\text { wire(single } \\
\text { sensor) }\end{array}$ & $\begin{array}{l}\text { Aerodynami } \\
\text { c noise }\end{array}$ & Axial fan & 2040 & 5 & 170 & 5.1 & 100 & 30 \\
\hline Read and Elder [3] & 1999 & $\begin{array}{l}\text { Hot-wire } \\
\text { (two } \\
\text { element) }\end{array}$ & $\begin{array}{l}\text { Turbulence, } \\
\text { length scales }\end{array}$ & $\begin{array}{l}\text { 4-stage axial } \\
\text { compressor }\end{array}$ & 1100 & 75 & 1375 & 58.7 & 384 & 43 \\
\hline $\begin{array}{l}\text { Prato and [6] } \\
\text { Lakshminarayana }\end{array}$ & 2001 & $\begin{array}{l}\text { Slanted hot- } \\
\text { film }\end{array}$ & $\begin{array}{l}\text { Unsteady } \\
\text { flow }\end{array}$ & $\begin{array}{l}\text { 3-stage axial } \\
\text { compressor }\end{array}$ & 5410 & 74 & 6672 & 133.4 & 250 & 20 \\
\hline $\begin{array}{l}\text { Velarde-Suárez et } \\
\text { al. [5] }\end{array}$ & 2002 & $\begin{array}{l}\text { Hot-wire } \\
\text { (triple } \\
\text { sensor) }\end{array}$ & $\begin{array}{l}\text { Total } \\
\text { unsteadines } \\
\text { s }\end{array}$ & $\begin{array}{l}\text { Low-speed } \\
\text { axial fan }\end{array}$ & 3000 & 8 & 400 & 12.8 & 80 & 32 \\
\hline $\begin{array}{l}\text { Huyer and Snarski } \\
\text { [1] }\end{array}$ & 2003 & $\mathrm{X}$ hot-wire & $\begin{array}{l}\text { Turbulent } \\
\text { inflow }\end{array}$ & $\begin{array}{l}\text { Axial } \\
\text { propeller }\end{array}$ & 600 & 6 & 60 & 10 & 49 & 167 \\
\hline Shiomi et al. [38] & 2003 & $\begin{array}{l}\text { Single } \\
\text { slanted hot- } \\
\text { wire }\end{array}$ & Internal flow & $\begin{array}{l}\text { Diagonal flow } \\
\text { fan }\end{array}$ & 1620 & 6 & 162 & 10.4 & 1500 & 65 \\
\hline $\begin{array}{l}\text { Mailach and } \\
\text { Vogeler [4] }\end{array}$ & 2004 & $\begin{array}{l}\text { Hot-film } \\
\text { (flush } \\
\text { mounted) }\end{array}$ & $\begin{array}{l}\text { Row } \\
\text { interaction, } \\
\text { boundary } \\
\text { layers }\end{array}$ & $\begin{array}{l}\text { 4-stage axial } \\
\text { compressor }\end{array}$ & 1000 & 63 & 1050 & 51.2 & 250 & 49 \\
\hline Jang et al. [39] & 2005 & $\begin{array}{l}\text { Three hot- } \\
\text { wire }\end{array}$ & $\begin{array}{l}\text { Tip leakage } \\
\text { unsteady } \\
\text { flow }\end{array}$ & $\begin{array}{l}\text { Low-speed } \\
\text { axial fan }\end{array}$ & 1200 & 9 & 180 & 10 & 108 & 56 \\
\hline Henderson et al. [9] & 2006 & Hot-film & $\begin{array}{l}\text { Turbulence, } \\
\text { wake } \\
\text { dispersion }\end{array}$ & $\begin{array}{l}\text { 1.5-stage } \\
\text { axial } \\
\text { compressor }\end{array}$ & 500 & 37 & 308.3 & 50 & 3072 & 162 \\
\hline Kergourlay et al. [2] & 2006 & $\begin{array}{l}\text { Hot fiber- } \\
\text { film (dual } \\
\text { sensor) }\end{array}$ & $\begin{array}{l}\text { Unsteady } \\
\text { flow }\end{array}$ & Axial fan & 2000 & 6 & 200 & 25 & 168 & 125 \\
\hline $\begin{array}{l}\text { Fernández Oro et } \\
\text { al. }[28,29]\end{array}$ & 2007 & X Hot-wire & $\begin{array}{l}\text { Turbulence, } \\
\text { length } \\
\text { scales, } \\
\text { unsteadines } \\
\text { s }\end{array}$ & $\begin{array}{l}\text { 1-stage axial } \\
\text { fan }\end{array}$ & 2400 & 9 & 360 & 36 & 100 & 100 \\
\hline Henner et al. [40] & 2007 & X Hot-wire & $\begin{array}{l}\text { Rotor-stator } \\
\text { interaction }\end{array}$ & Axial fan & 3000 & 9 & 450 & 30 & 275 & 67 \\
\hline Vad et al. [41] & 2007 & $\begin{array}{l}\text { Cross hot- } \\
\text { wire }\end{array}$ & $\begin{array}{l}\text { Aerodynami } \\
\text { c effects }\end{array}$ & Axial fan & 416 & 12 & 83.2 & 10 & 104 & 120 \\
\hline
\end{tabular}




\begin{tabular}{|c|c|c|c|c|c|c|c|c|c|c|}
\hline Lepicovsky [7] & 2008 & $\begin{array}{l}\text { Split fiber } \\
\text { hot-wire }\end{array}$ & $\begin{array}{l}\text { Passage flow } \\
\text { field }\end{array}$ & $\begin{array}{l}\text { 4-stage axial } \\
\text { compressor }\end{array}$ & 984 & 39 & 639.6 & 10 & 65 & 16 \\
\hline $\begin{array}{l}\text { Eberlinc et al. } \\
{[42,46]}\end{array}$ & 2009 & Single wire & $\begin{array}{l}\text { Trailing edge } \\
\text { blowing }\end{array}$ & $\begin{array}{l}\text { Hollow- } \\
\text { blade, axial } \\
\text { fan }\end{array}$ & 1130 & 7 & 130 & 50 & 780 & 375 \\
\hline Shiomi et al. [43] & 2009 & $\begin{array}{l}\text { Single slant } \\
\text { hot wire }\end{array}$ & $\begin{array}{l}\text { Unsteady } \\
\text { flow }\end{array}$ & $\begin{array}{l}\text { Swept blade, } \\
\text { semi-open } \\
\text { fan }\end{array}$ & 3550 & 5 & 295 & 20 & 300 & 68 \\
\hline Hurault et al. [44] & 2010 & $\begin{array}{l}\text { Hot fiber- } \\
\text { film (dual } \\
\text { sensor) }\end{array}$ & $\begin{array}{l}\text { Unsteady 3D } \\
\text { flow }\end{array}$ & $\begin{array}{l}\text { Sweep blade } \\
\text { axial fan }\end{array}$ & 2700 & 8 & 360 & 45 & 160 & 125 \\
\hline Sarraf et al. [45] & 2011 & $\begin{array}{l}\text { Dual hot } \\
\text { wire }\end{array}$ & $\begin{array}{l}\text { Blade } \\
\text { thickness } \\
\text { effects }\end{array}$ & $\begin{array}{l}\text { Vortex- } \\
\text { designed } \\
\text { axial flow fan }\end{array}$ & 2000 & 6 & 200 & 120 & 900 & 600 \\
\hline
\end{tabular}

\section{2.- DATA REDUCTION TECHNIQUES}

Phase-locked averaging (PLA) technique. Phase-locked averaged values in turbomachinery environments are computed by isolating a number of blade passages, dividing them into a fixed number of classes, and making averages among the corresponding classes $(\ldots k-1, k, k+1 \ldots)$. The number of classes is chosen as the number of samples recorded per blade passing event. This ensemble-averaging technique samples a time series of data obtained over many rotor revolutions based on the phase or angular position of a rotor blade. A signal from a shaft encoder is typically used for synchronizing the sensor time series with the phase of the machine [20].

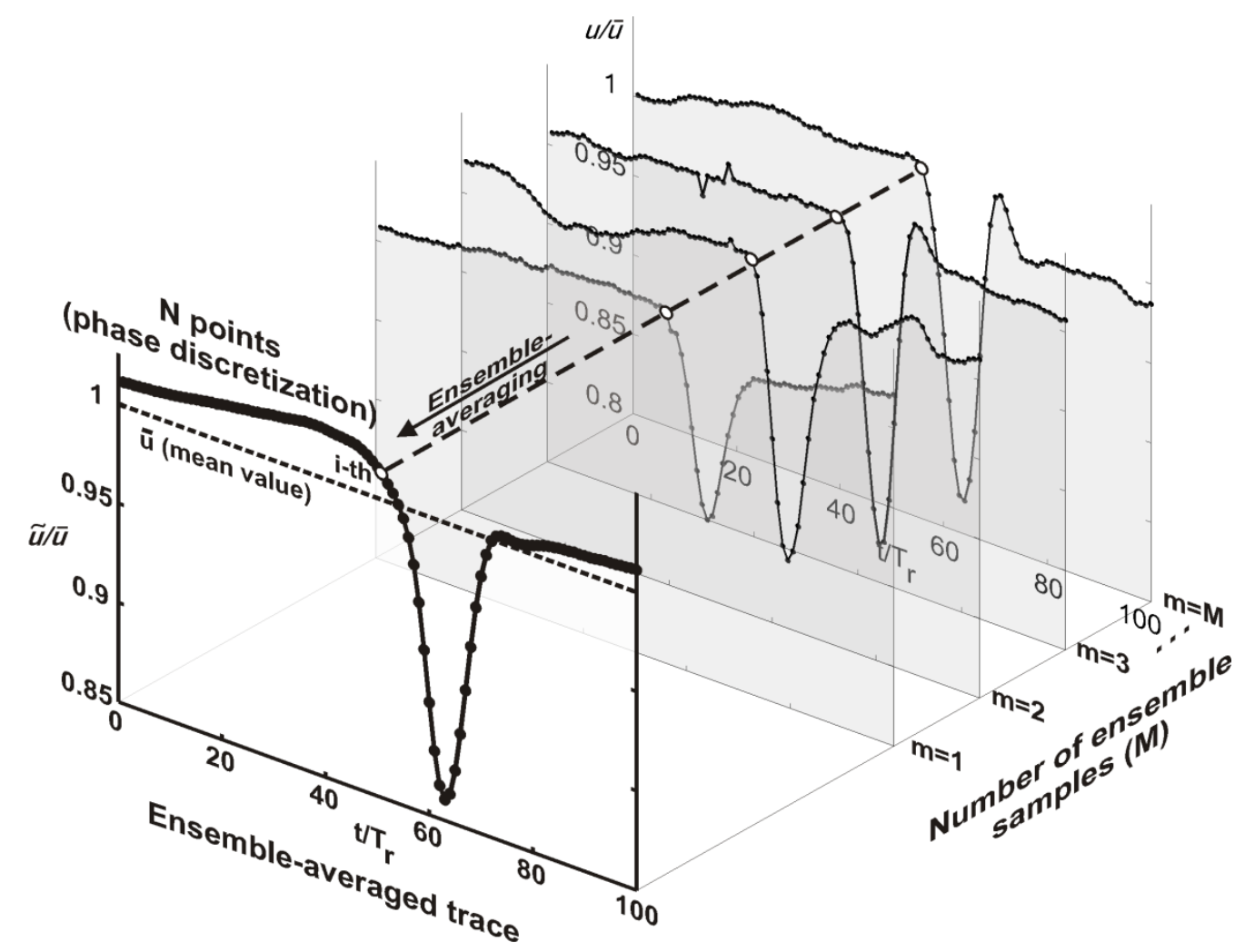

Figure 1. Phase-locked averaging technique. 
For data recorded over $M$ blade passing events with a stationary probe, considering periodicity between consecutive rotor blades, the phase- or ensemble-averaged value of a trace velocity component $\mathrm{u}$ at time or phase $\mathrm{n}$ (angular position) is obtained as:

$$
u_{n}^{(M)}=\frac{1}{M} \sum_{m=1}^{M} u_{n}^{(m)}
$$

where the subscript $n$ represents the $n$-th angular position of the measured trace and the superscript $(M)$ indicates that the ensemble-averaging has been completed using $M$ realizations. The superscript symbol represents the ensemble-averaging. Figure 1 illustrates this methodology to calculate ensemble-averaged data using continuous time series.

Additionally, non-deterministic scales (turbulence) are directly obtained subtracting the timeresolved (or phase-resolved) velocity defined in (1) from the original, instantaneous trace, according to $u_{n}^{\prime}=u_{n}-0 \theta_{n}^{\prime M)}$. This turbulent component results in a raw signal that needs further processing to provide a statistical meaning. Hence, the level of non-deterministic unsteadiness can be estimated applying also the ensemble-averaging:

$$
\vec{d}_{n}^{(2)}=\frac{1}{M} \sum_{m=1}^{M}\left[u_{n}^{(m)}-Q d_{n}^{(M)}\right]^{2}
$$

where the phase dependency (subscript $n$ for every angular position of the tangential displacement of the blade measured with the stationary probe) reveals the transport of the ensemble, non-resolved structures in the unsteady, deterministic flow patterns.

Furthermore, the turbulence intensity is defined from equation (2) to express the "strength" of the turbulent motion. It is simply defined as the root mean square (RMS) of these ensembleaveraged fluctuations, and divided by the ensemble velocity (1) in order to obtain the unsteady turbulent field (for $M$ ensembles), thus resulting:

$$
T u_{n}^{(M)}=\frac{\sqrt{\frac{d_{n}^{(M)}}{d_{n}^{(M)}}}}{e_{n}^{\delta^{(M)}}}
$$

Finally, it is possible to obtain a time-averaged velocity and a time-averaged turbulence level (mean turbulence), introducing a time-averaging (or phase-averaging) operator that provides the overall (steady) mean value of variables (1) and (3) for a blade passing period. Here, the overbar denotes the time-averaging:

$$
\bar{u}=\frac{1}{N} \sum_{n=1}^{N} \theta \theta_{n}^{(M)} \quad ; \quad \overline{T u}=\frac{1}{\bar{u}} \sqrt{\frac{1}{N} \sum_{n=1}^{N} \vec{d}_{n}^{(2)}}
$$

At this point, it must be advised that it is necessary to perform a frequency domain analysis in the first stage of the post-processing routines. Here, the starting point is to check for the periodicity of the studied phenomena, so it can be assured that the phase-averaging is meaningful. Typically, the use of digital filters is highly recommended, especially beyond the frequency 
response of the instrumentation. Secondarily, it is possible to find harmonics at low frequencies that are deterministic but uncorrelated with the blade passing frequency (Goto [21] or Fernández Oro et al., [22]). For this reason, the signals should be first analyzed in the frequency domain to highlight potential sources of noise, and then filtered in a proper way to be finally phased-lock averaged. Alternatively, possible development of non-periodical fluid-dynamical phenomena can be highlighted by a non-gaussian probability density distribution of the experimental values (Cavazzini et al., [23]). Therefore, another useful method is the analysis of the probability density distribution of the determined velocity values (Heinz et al., [24]). In summary, these recommendations suggest that the statistical analysis should be completed with a verification of the supposed periodicity.

It is evident from equations (2) and (4) that the accurate selection of the number of ensembles $(M)$ and the number of samples $(N)$ per blade event is crucial to obtain a reliable statistical description of the time-resolved traces. As both values are progressively increased, the timeresolved evolution is clearly enhanced, reducing inaccuracies and achieving overall convergence (figure 2). Particularly, in the case of the number of ensemble averages, turbulence is removed and periodic (deterministic) unsteadiness is recovered when a sufficient number of ensembles is performed. Figure 2(a) illustrates how for a typical trace, at least 80 ensembles are necessary for converged statistics in that case. On the other hand, the larger the number of samples, the higher the tangential accuracy is. Hence, if a quite reduced number of points is fixed (due to a low sampling frequency), then the trace will be badly-discretised, as it is shown in figure 2(b) for traces sampled with juts 10 or 20 points. In that plot, it is clear that the same trace is well-described only if 75 or 90 points are at least employed.

Ensemble averaging (M samples for statistics)

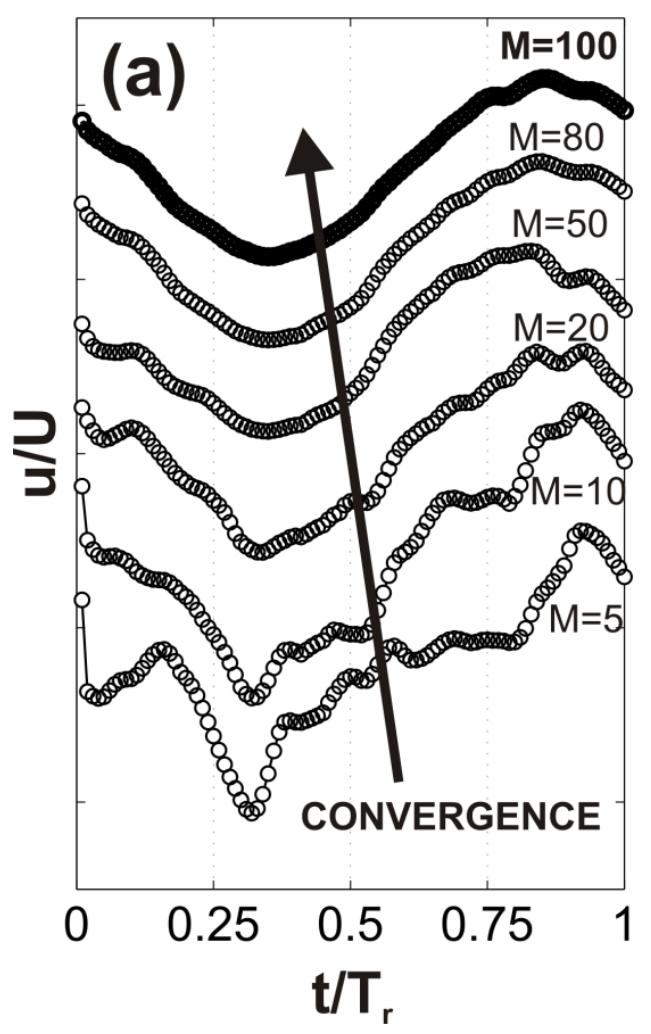

Sampling frequency

( $\mathrm{N}$ samples for accuracy)

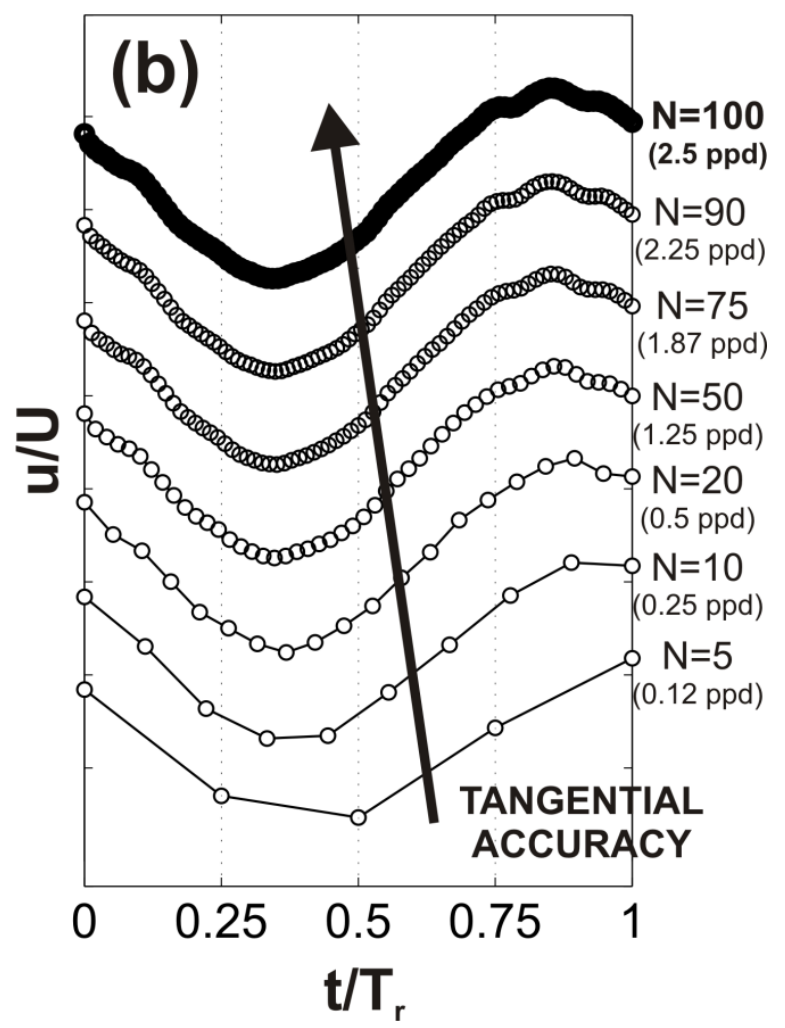

Figure 2. Convergence (a) and tangential accurary (b) for time-resolved traces. 
Convergence of the ensemble-averaging operator. Because the number of ensemble averages needed to assure accurate time-resolved structures is not known a priori, it is necessary to define a statistical indicator that could provide a minimum required number within a confidence level. Obviously, in most situations, the final trace is what we are precisely trying to measure, so this indicator cannot be defined through a classical error definition with respect to the true value. Instead, the total number of samples adopted must be analyzed in terms of convergence, introducing a residual $\mathrm{R}$ as the RMS value of the difference between the time-resolved traces using $M$ and $M-1$ ensembles for the total number of points defining the blade event. This definition is divided by the averaged value of the trace as a scaling factor, in order to define a scaled residual, which is a more appropriate indicator of convergence. Mathematically, it can be expressed as:

$$
R=\frac{\sqrt{N \sum_{n=1}^{N}\left(\dot{\theta}_{n}^{M)}-\dot{\theta}_{n}^{M-1)}\right)^{2}}}{\sum_{n=1}^{N} \theta \theta_{n}^{M)}}
$$

Typically, residuals are shown in logarithmically-scaled plots, with a default convergence criterion usually fixed to $10^{-3}$, in analogy to CFD practices. Note that this definition of residual is a sort of a relative error difference, comparing local (point-by-point) discrepancies between two consecutive traces on the convergence process. In essence, it monitors the global shape of the trace, giving a measure of the relative change as the number of ensembles is increased and stopping when this change is lower than a $0.1 \%$.

Figure 3(a) illustrates the evolution of the residual after being applied over the ensemble averaging process previously shown in figure 2(a). Typically, the residual is rapidly decreased for a few numbers of ensembles to be slowed down once it falls below $10^{-2}$. The figure also points out the residual levels calculated for the ensemble traces shown in figure 2 , highlighted with white dots in the plot. In this case, the typical criterion $\left(10^{-3}\right)$ is not met even with a hundred ensembles, so a higher number of recorded blade events would be required to achieve such a convergence threshold. However, from the traces shown in figure 2, it is quite presumable to admit that such restrictive levels are quite excessive, and that a more reasonable threshold of $5 \cdot 10^{-3}$ could be perfectly adopted in this case.

Intuition dictates that the more turbulent or the more disturbed the velocities traces are, the larger number of ensembles will be necessary to filter out the turbulence effects. Effectively, taking advantage of the definitions introduced by the phase-locked averaging technique, it is possible to reformulate the quadratic difference of the numerator in equation (5) as a function of the overall mean turbulence of the measured traces. As a consequence, an estimation of the minimum number of ensemble averages required to obtain a statistically accurate description of the flow magnitude can be also expressed as a function of the fluctuation (the local turbulence level) for a desired convergence criterion according to:

$$
M \cong \frac{1}{R} \overline{T u}
$$

Derivation of this expression is shown in detail in annex A. Note that for a typical total unsteadiness in the order of an $8 \%$, with the usual $10^{-3}$ limit, the number of required ensembles should be just around 80 . 

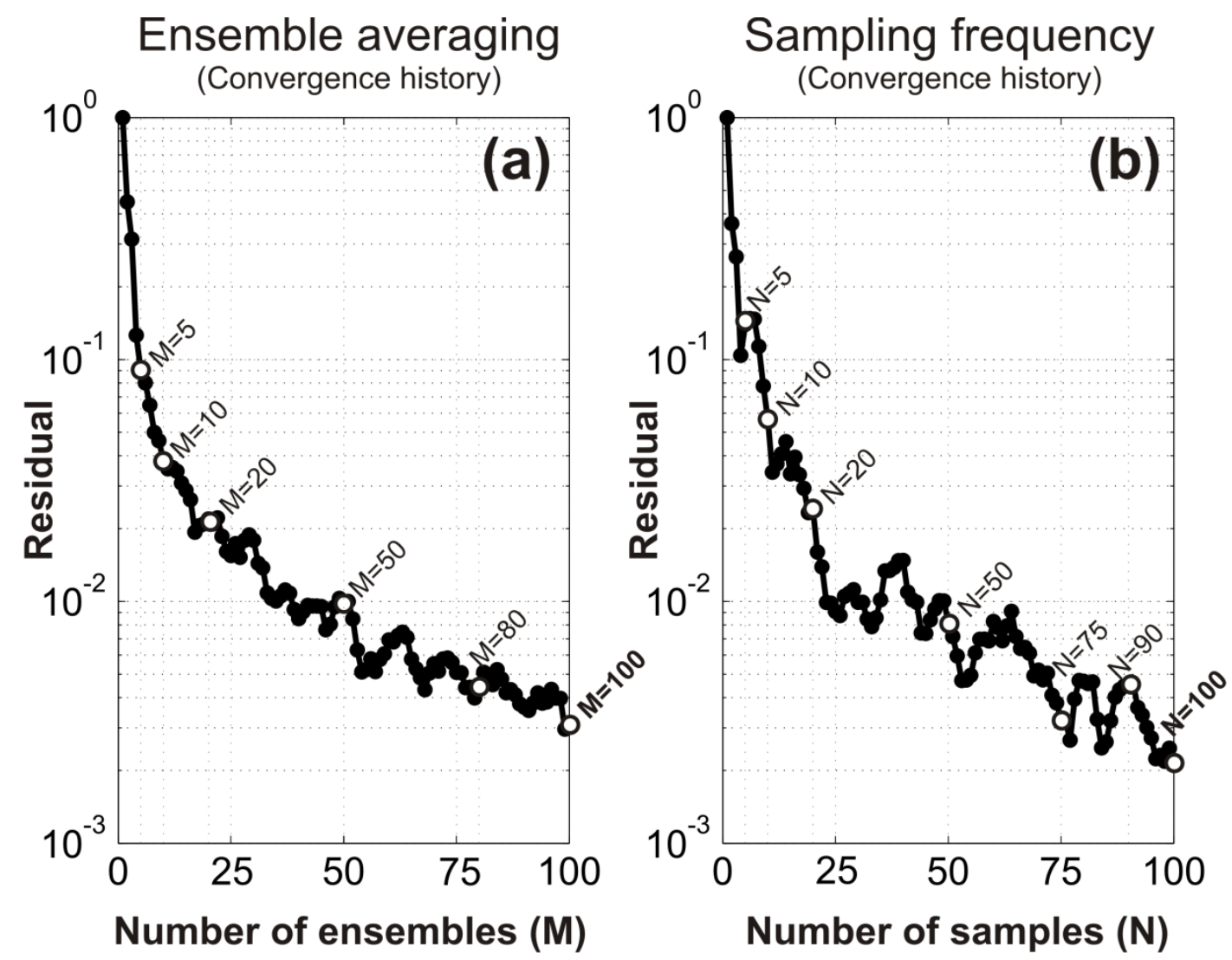

Figure 3. Convergence histories for ensembling (a) and sampling (b) operators.

Tangential accuracy for the number of samples per blade event. In the case of the number of samples required to define a sufficient tangential accuracy, the residual indicator has been again introduced because of its suitability to compare the relative change in the whole shape of the traces. It must be advised that to compute the residual between traces with $n$ and $n-1$ samples it is necessary to interpolate the trace with less number of points to the elements defining the $n$ pointed trace. Basically, the residual tracks the evolution of the time-resolved signal with increasing discretization points and, in the case of stationary probes, it accounts for the precision of the tangential blade-to-blade gradients.

The representation of this residual as a function of the number of circumferential points used to describe the blade passing (or blade event) is analogous to determine the impact of the points per degree selection over the velocity tangential gradients in the relative reference frame. In the case of turbomachinery, the number of points per degree depends directly on the passage angle, that is, the whole 360 deg of the circumference divided by the number of rotor blades. Remember that typical values of points per degree used in the literature present large discrepancies, ranging from 0.75 to roughly 9.00 due to the flow structures and velocity fluctuations in every case.

Figure $3(b)$ shows the convergence history for the residual of the example trace as a function of the number of samples, using now the maximum available number of ensembles. Corresponding values of residual with respect to under-converged traces shown in figure 2 have been also identified with white dots. Note that the behavior of this indicator with respect to the number of samples, $N$, is similar to that already discussed for the number of ensembles, $M$.

As in the case of turbulence as a critical factor for the convergence of the ensemble averaging operator, irregularities and fluctuations of the time-resolved traces play now a major role in the determination of the number of sample points needed for a satisfactory tangential accuracy. If the 
blade-to-blade gradient is extremely irregular, then a huge number of elements is required to provide a well-defined description of the velocity pattern.

In order to explore the relationship between convergence criteria and usual gradients associated to typical wake profiles found in axial turbomachinery, a theoretical wake velocity profile has been considered for the discussion:

$$
\frac{U-U}{U}=\frac{D}{2}\left[1+\cos \left(\frac{2 \pi y}{\delta}\right)\right]
$$

where $D$ represents the wake velocity deficit (or wake depth), $\delta$ is the wake width and $U$ represents the maximum value out of the deficit. This velocity profile has been previously employed in the literature as representative of rotor wakes in axial compressors [25, 26]. Using this theoretical trace (normalized for both depth and width dimensions), the definition for the residual has been employed to determine the minimum number of samples (discrete points) needed to reproduce the mathematical formulation so the wake profile could be recovered in a discrete form within a convergence limit of $5 \cdot 10^{-3}$. The results, as well as a schematic representation of the wake profile, are shown in figure 4, for several deficits of the wake (ranging from 0.2 to 0.9 ), and typical wake widths (going from 0.05 to 0.5 , according to Greitzer et al. [27]).
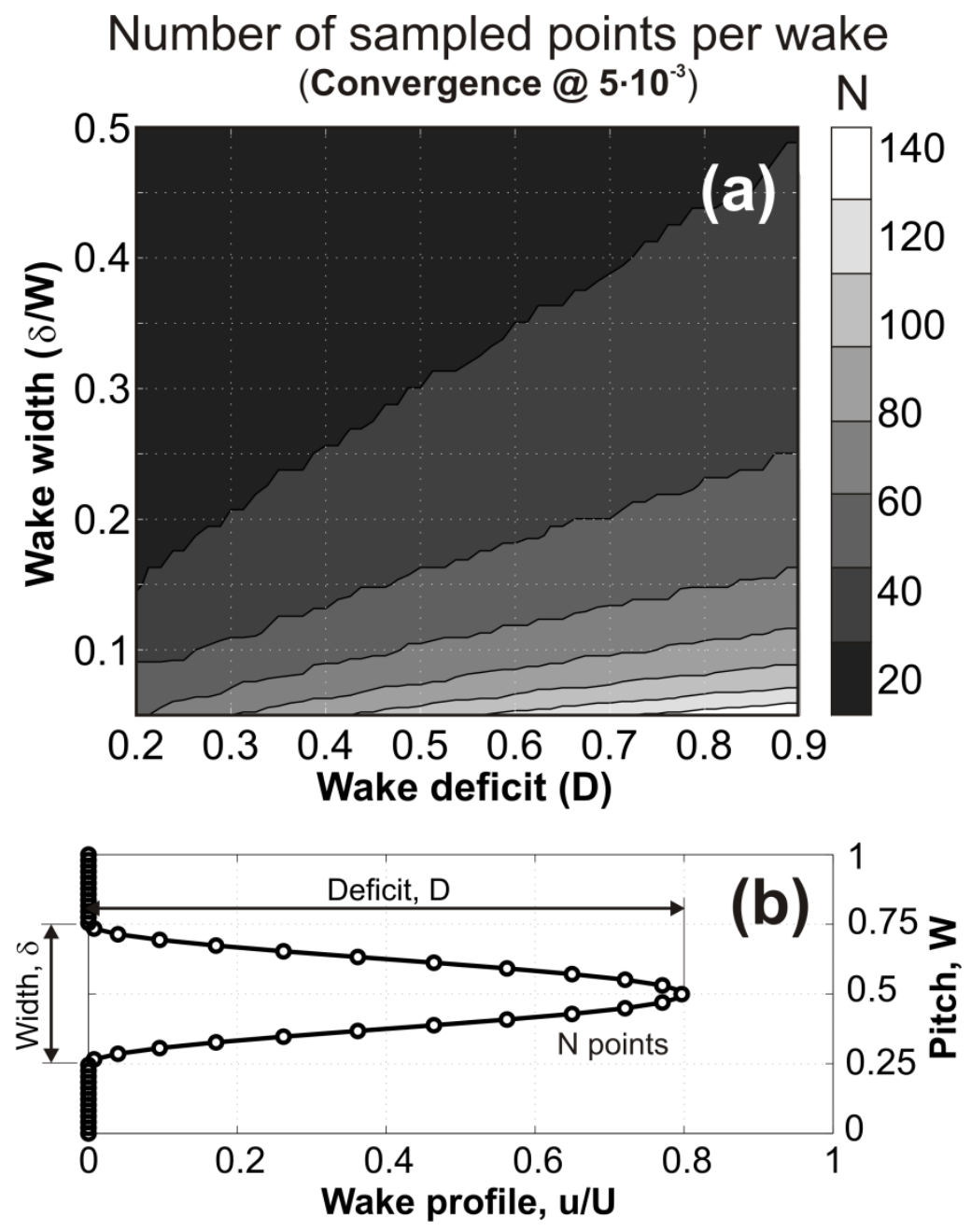

Figure 4. Influence of the wake profile in the number of sample points required for a converged tangential accuracy. (a) Minimum number of samples. (b) Wake profile. 
There is a notable difference between the number of points (around 125) required for narrow and sharp wakes (bottom right corner) and the number (approximately 25) estimated for thick, blunt profiles (top left corner), thus revealing the evident influence of the wake shape in the number of samples required. Moreover, if the non-wake region would be also irregular, instead of a plateau as in the theoretical profile, then the number of points should be surely increased. Additionally, if other unmixed wakes coming from previous stages in case of multi-rows environments may be contributing in the velocity trace; or secondary flows (like tip vortex, wakewake interactions,...) could arise in the velocity measurements, then the number of points would be even higher. Consequently, we can expect that for this type of traces, a typical number of a hundred of samples is a reasonable order of magnitude to evaluate blade-to-blade gradients in an efficient way. The corresponding points-per-degree for the sampling frequency will depend on the extension of the passage pitch, that is, on the number of rotor blades involved.

The consideration of a theoretical profile allows computing the real error that it is made due to the representation of the wake in a discrete form. In particular, it is a common practice to characterize this kind of wake distributions using their integral parameters, like the displacement thickness or the momentum thickness. In this example, we have employed the displacement thickness that can be considered as the first moment of the vorticity that accounts for the mass deficit carried in the boundary layer:

$$
\frac{\delta^{*}}{W}=\frac{1}{W} \int_{-\delta / 2}^{\delta / 2}\left(1-\frac{u}{U}\right) d y
$$

where direct analytical integration of the cosine law for the wake results in a displacement thickness equal to $\delta^{*}=D \delta / 2$. Consequently, we can compute the error associated to the displacement thickness $\left(\delta_{p}^{*}\right)$ given by the area under the curve of the trace sampled with the number of points meeting the convergence criterion (given in figure 4), with respect to its exact value $\left(\delta^{*}\right)$, that is:

$$
e(\%)=100 \cdot\left|\delta_{p}^{*}-\delta^{*}\right| / \delta^{*}
$$

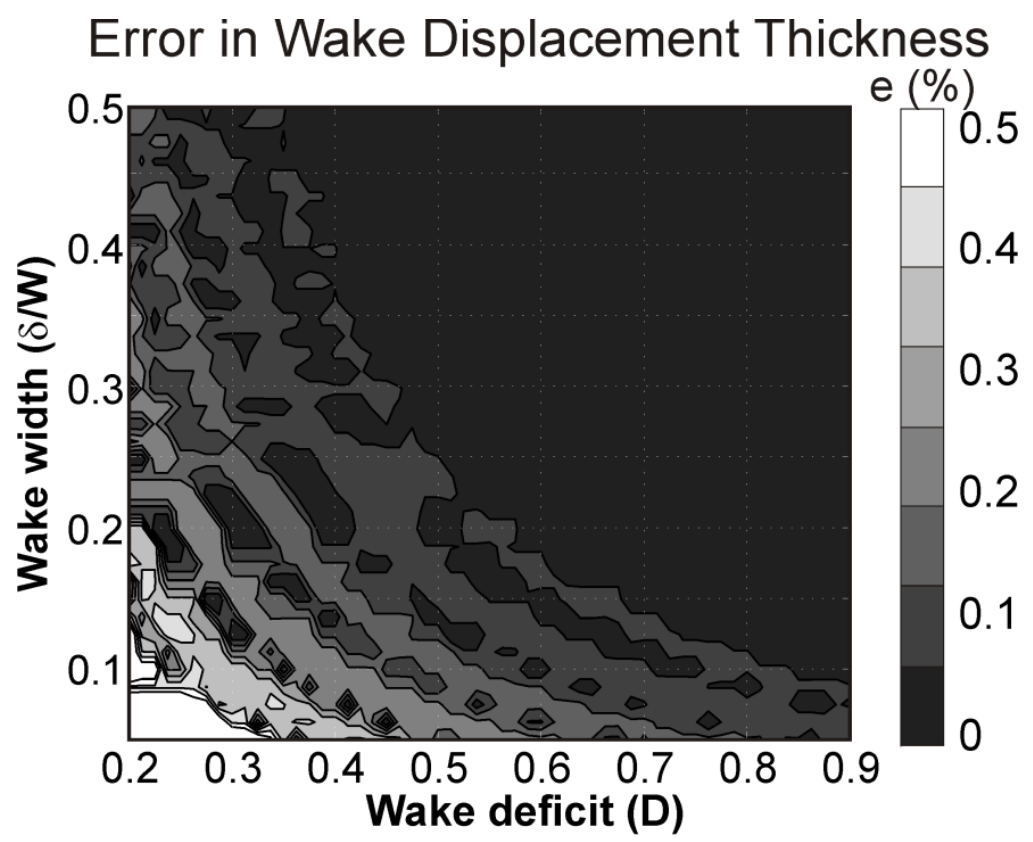

Figure 5. Estimation of the error in the wake displacement thickness for a wake profile discretized with $\mathrm{N}$ points given a minimum residual criterion of $5 \cdot 10^{-3}$. 
The results are plotted in figure 5. Except for a certain diffusion in the data, it is evident that a convergence criterion of $5 \cdot 10^{-3}$ provides maximum errors of only a $0.5 \%$, especially in the case of very thin wakes with extremely low deficits. As a consequence, whatever the case, it is quite clear that with these results, the discrepancies are totally assumable and the number of samples adopted is really accurate. Though not shown here, similar values (and distributions of error respect the wake deficit and width) have been obtained when comparing the momentum thickness (second moment) or the energy thickness (third moment).

In summary, the residual indicator is presented here as a useful tool to estimate the final precision of our experimental measurements. Additionally, it has been clearly demonstrated its ability to provide both suitable ensemble and sample parameters. Following, this formulation will be employed in an application database concerning a single-stage, low-speed axial fan with twin configurations: rotor-stator and stator-rotor arrangements, correlating convergence maps with turbulent structures measured within the stator-rotor gap and also at the machine discharge.

\section{3.- APPLICATION FOR A SINGLE-STAGE, LOW-SPEED AXIAL FAN}

Measuring database. An experimental database has been obtained using dual hot-wire anemometry (classic X-type probe) across the stage of a single-stage low-speed axial fan with both stator-rotor (S-R) and rotor-stator (R-S) configurations. This axial blower consists of a 9-bladed rotor with a 13-vaned stator operated at $2400 \mathrm{rpm}(\mathrm{BPF}=360 \mathrm{~Hz})$ and tip and hub diameters of 820 and $380 \mathrm{~mm}$ respectively. The design conditions for the fan are $16.5 \mathrm{~m}^{3} / \mathrm{s}$ of delivered flow rate and $1.2 \mathrm{kPa}$ of pressure rise. The anemometric probe, a 120 deg crossed X-dual hot wire, with an estimated frequency response of the wires in the range of $20-30 \mathrm{kHz}$, was intensively employed rotor downstream and within interrow regions in a number of traverse measuring windows of stator pitch periodicity - see figure 6(a) . The radial discretization of the windows ranged from 15 to 23 spanwise locations, whereas the tangential discretization ranged from 15 to 28 pitchwise positions - see figure 6(b). Nominal (Qn) and off-design (70\% Qn) conditions have been analyzed, allowing the study of the influence of flow distortion on convergence parameters. More geometrical and operational details can be found in [28].

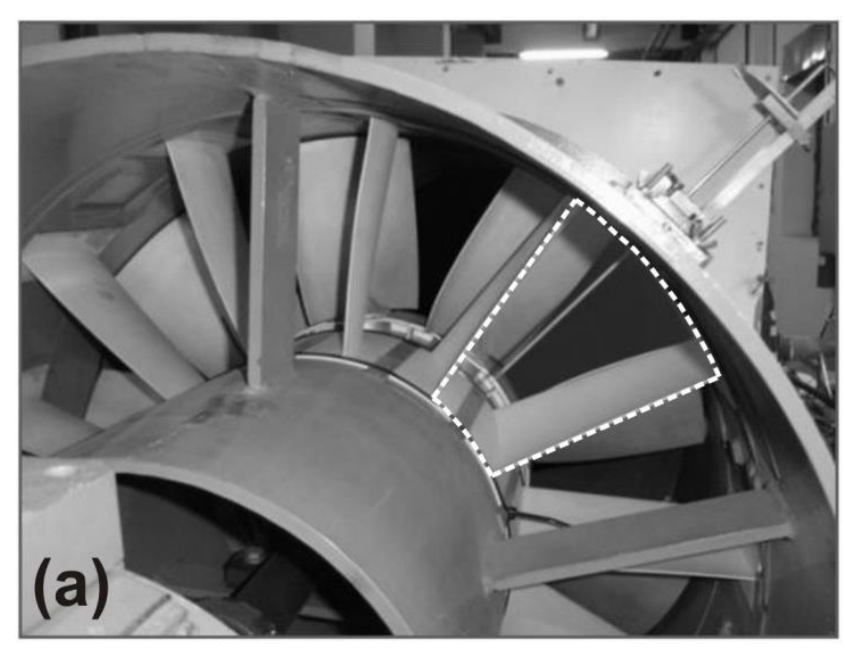

Experimental facility. Traverse windows (DHW probe at measuring location)

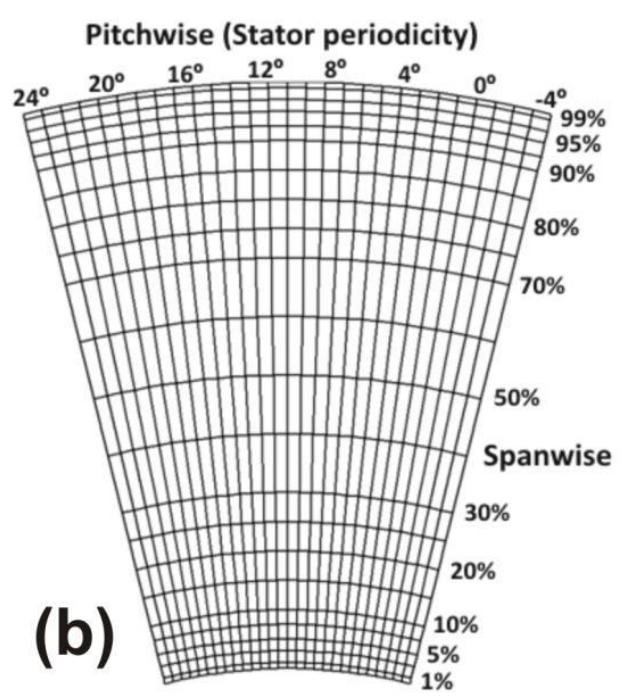

[23×29] spatial resolution

Figure 6. Measuring windows (a) and spatial discretizations (b). 
Concerning acquisition parameters, a temporal resolution of $N=100$ samples per blade passing period was fixed, resulting in a sampling rate of $36 \mathrm{kHz}$ (a hundred times the BPF) and 2.5 points per degree. Also, for every velocity trace, up to $M=200$ blade passing periods ( 22 revolutions for a 9-bladed rotor at $2400 \mathrm{rpm}$ ) were approximately recorded, so a total number of $2 \cdot 10^{4}$ samples were stored during $0.544 \mathrm{~s}$ at every measuring location. In the case of R-S configuration an additional test was completed running the axial fan at $1200 \mathrm{rpm}$, so the number of samples per blade passing was also extended to $N=200$, that is, 5 points per degree.

Post-processing routines. A preliminary analysis of the measured traces has been performed in the frequency domain in order to check the periodicity of the flow structures and verify the suitability of the assumed hypothesis. The instantaneous signals have been low-pass filtered using a typical 5th-order Butterworth filter with a $6 \mathrm{kHz}$ cut-off frequency. Following, the power spectral density of the input signal is obtained in a number of selected positions using Welch's averaged modified periodogram, segmented into eight sections of equal length, each with $50 \%$ overlap, and windowed with a classic Hamming window. Representative results of the database are shown later in section 4. In addition, maps of turbulence intensities have been obtained in different interrow/outlet sections of the low-speed axial fans using the metrics defined in section 2 of the paper. Similar techniques of digital filtering were employed in this case for signal preconditioning.

\section{4.- RESULTS AND DISCUSSION}

A first look on convergence in velocity measurements stage downstream. As a starting point, the convergence of the measurements stage downstream are analyzed for both velocity magnitude and flow angle retrieved with the X-probe in the R-S configuration. Figure 7 illustrates the degree of convergence as a function of the number of ensemble averages, ranging from 2 to 200, with a default convergence criterion of $10^{-3}$. Solid lines represent nominal conditions, whereas dashed lines show results from off-design working points. In figure $7(a)$, results from midspan regions (free of wall-annulus shear layers) are represented, whereas in figure $7(b)$, characteristic evolutions within boundary layers are reported for comparison.

The distributions reveal how it is necessary a larger number of ensembles in the case of disturbed regions of the flow (convergence is clearly slowed down in the figure 7 (b). In particular, in the represented case for the hub boundary layer, neither the flow angle nor the velocity residuals met the default convergence criteria within the maximum limit of $M=200$ ensembles of the database. Additionally, the phase-averaged flow angle presents the slowest convergence history, seriously affected by the operating conditions and even quite insensitive to increments in the number of ensemble averages. This may be caused by the zero mean value of the outlet flow angle for the single axial stage, which implies that small misalignments of the instantaneous flow with respect to the streamwise direction are perceived as important instabilities. This effect is dramatic at off-design conditions, in the near-stall region of the performance curve, with higher separation, wake instabilities and generation of large-scale turbulence. Flow turns instable in the rotor wake region and the ensemble-averaging procedure lacks fidelity due to the own nature of the discharge flow. 


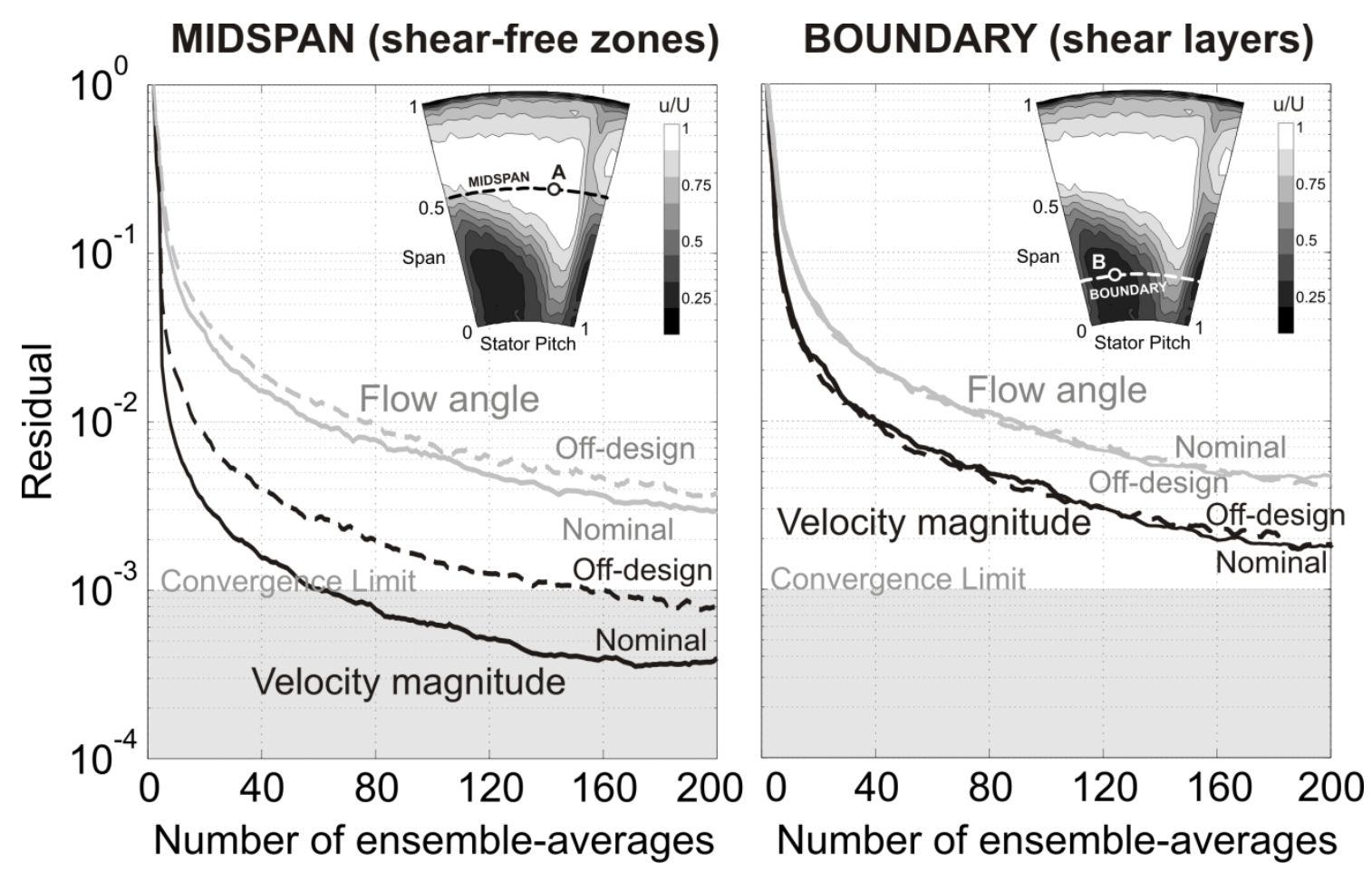

Figure 7. Residual histories for velocity and flow angle, stage downstream of the R-S configuration. Influence of the operating conditions at midspan (a) and boundaries (b) for the ensemble convergence.

On the contrary, the convergence for velocity magnitude is perfectly met at both nominal and off-design conditions when 60 and 150 ensemble-averages are respectively used at midspan. This suggests expected turbulence intensity in the range of a $6-15 \%$ for stator downstream locations at midspan (equation 6). Effectively, the authors have reported in previous investigations [29] overall mean levels that are totally in concordance with these estimations coming from the statistics. Note that at off-design conditions, the overall flow disorder affects significantly the spanwise convergence, compromising the fixed criterion.

A first look on tangential accuracy in measurements rotor downstream. The S-R configuration has been employed to analyze the required sampling frequency that it is desirable to obtain a converged blade-to-blade description of the flow. Thus, results on convergence of that tangential discretization have been resumed in figure 8 , following the same conventions as before. Similar conclusions and behaviours are also appreciable for this second indicator of accuracy.

At end-wall boundaries, the instabilities arise and the oscillations of the wakes require a larger number of samples per blade event. Here, the most important characteristic is that off-design conditions need to be described with a less number of ensembles because off-design flow patterns involve thicker wakes with lower velocity deficits [28], traceable with less points than narrow and peaked wakes at nominal operation. However, only the evolutions for the velocity at midspan are capable to meet the convergence threshold with less than 200 samples, or in other words, with less than 5 points per degree (grey $x$-axis in the plot). Note that the election of $N=200$ points to cover the blade displacement implies that both pressure and suction sides of the rotor wakes have been sampled with at least 20 points, in the order of typical discretizations employed in the literature [30]. 


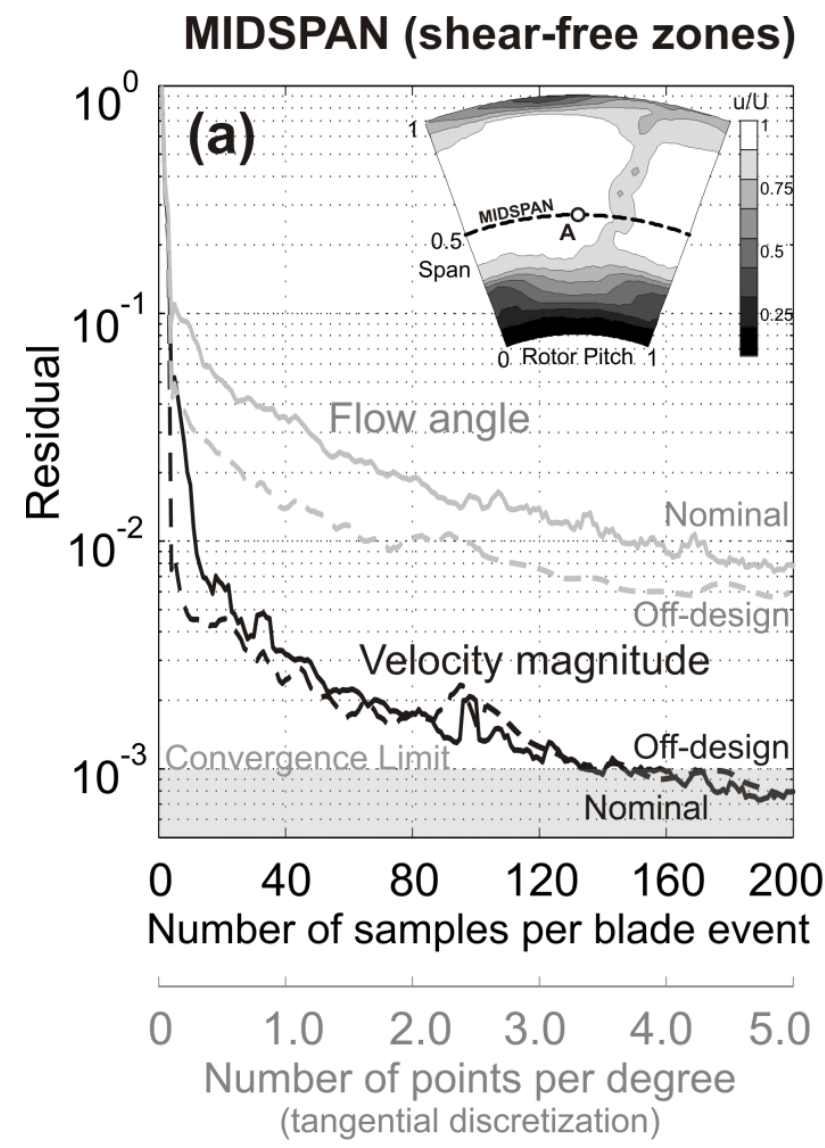

\section{BOUNDARY (shear layers)}

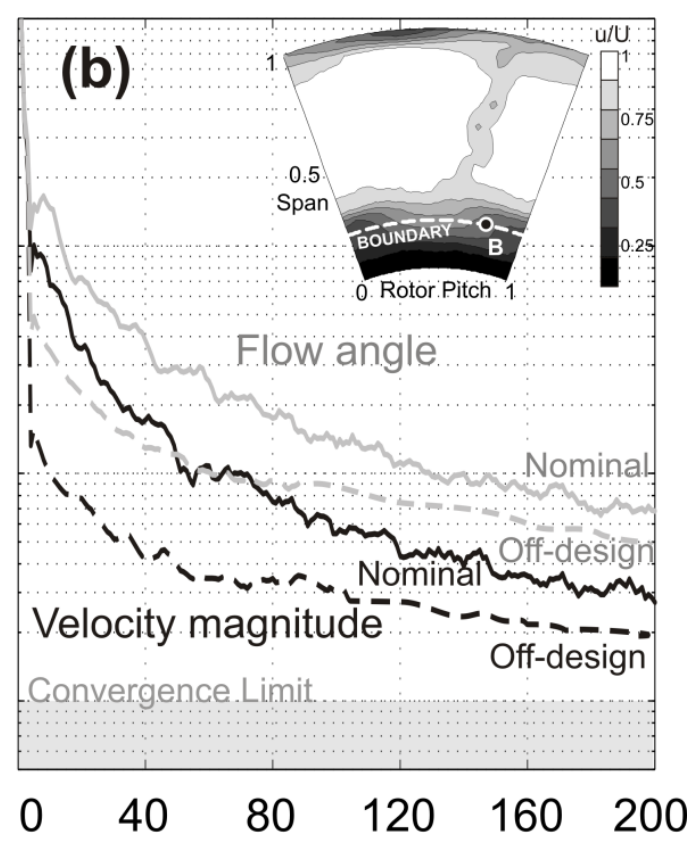

Number of samples per blade event

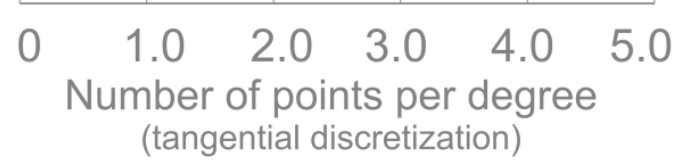

Figure 8. Residual histories for velocity and flow angle, rotor downstream of the S-R configuration. Influence of the operating conditions at midspan (a) and boundaries (b) for the samples convergence.

On the periodicity of the measurements throughout the stage. This first approach to the converged statistics of representative locations within the stage must be completed with the frequential analysis of the signals. The periodicity of the studied phenomena must be clearly observed, so the hypothesis of unsteady events clocked with the blade passing frequency could be verified. Figure 9 shows the power spectrum density (PSD) for the fluctuating component of the streamwise velocities at the same representative points analyzed in figures 7 and 8 . The same digital low-pass (6 kHz cut-off), 5th-order Butterworth filter for preconditioning, and parametric, $50 \%$ overlap, Welch periodogram for power spectra estimation have been employed here. Rotor downstream -figure 9(a)- the wake structure at midspan is perfectly observed in the periodogram (black spectrum) with the signature of the BPF $(0.36 \mathrm{kHz})$ and its harmonics (up to the ninth one), characteristic of narrow and sharp velocity deficits. In the boundary region, only the fundamental peak is recovered, indicative of a blunt and wide velocity fluctuation (rotor wake broadens within the endwall turbulence). Figure 9(b) shows similar spectra stage downstream where wake-mixing is rapidly taking place. As a consequence, even at midspan locations, the BPF signature is clearly weakened, whereas in boundary regions its presence is almost marginal. Also notice how the baseline spectra (i.e., the turbulent component if the peaks are removed) at the boundaries are always above those corresponding to the midspan locations because turbulence intensity is obviously higher in the shear regions. Whatever the case, in the vicinity of the rotor wakes for the locations of interest, the periodicity of the signals is clearly correlated with the BPF, thus validating the assumed hypothesis. 

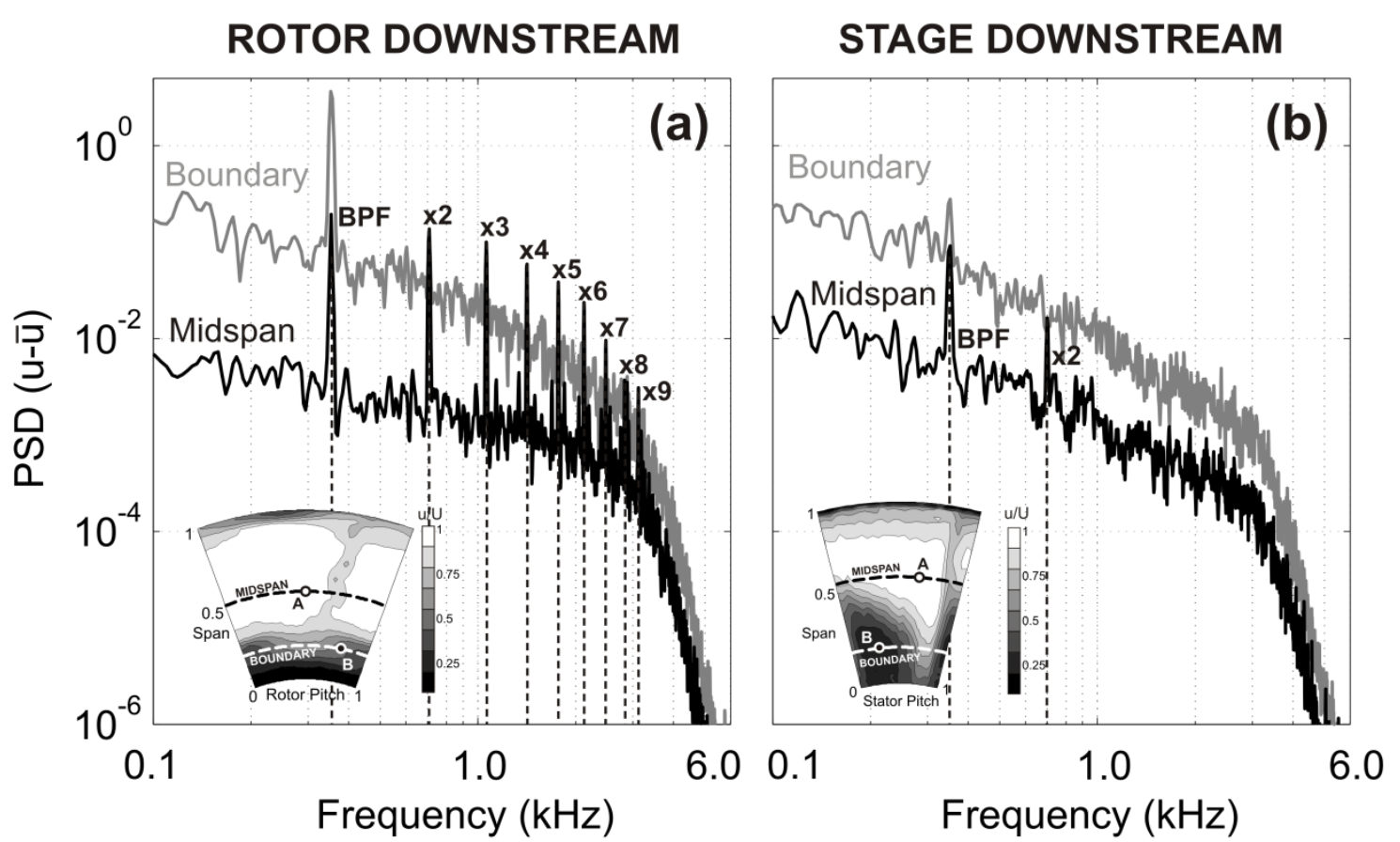

Figure 9. Power spectrum density for instantaneous velocity signals, rotor downstream of the S-R configuration (a) and at stage downstream (b) for midspan and boundary locations.

Impact of flow structures on number of ensembles. Following, the evolution with the spanwise location in the traverse measurements is also explored in order to advance on the impact of the shear flows in that convergence criteria. For that purpose, we have evaluated the number of ensemble-averages needed to assure convergence (fixed to $10^{-3}$ for S-R and $2 \cdot 10^{-3}$ for R-S) for the entire measurement grids of the database. Figure 10 shows in a two-column layout the results obtained for both nominal and off-design flow rates.

On the one hand, in the case of the S-R configuration -figures 10(a) and 10(b)- , midspan locations present quite stable flow patterns, allowing a moderate number of ensembles (in the range of 25-35) to converge. The circumferential uniformity is broken up by the presence of an unmixed wake coming from the upstream stator (already reported in [28]). In the presence of that wake, the required value is increased to almost 50 samples in order to obtain an accurate statistically reduction of the turbulent wake shear layers. Moreover, in both tip and hub boundary layers, the instability induced by the continuous generation of turbulence implies that a large number (>100) of ensembles should be employed to obtain reliable results. This increase in the number of samples must be also related to the major disorder of the flow and the averaged effect of the three-dimensionality of endwall structures induced by the rotor blade displacement (tip vortex, hub flow separation, etc...). Complementarily, results at off-design flow rates -figure 10(b)present analogous conclusions. In this case, the central region where the flow is more stable has been severely reduced. The contribution of the unmixed stator wake is still visible, with a significant thickening of the wake width that requires a larger number of ensembles. Similarly, the boundary layers have been dramatically engrossed, especially in the tip region, indicating the major flow disorder of the turbulent structures at near-stall conditions. Note that contour maps at this extreme positions is saturated, indicating that more than 100 ensembles are needed to devise a $10^{-3}$ convergence.

On the other hand, the R-S configuration -figures 10(c) and 10(d)- presents a completely different pattern as a consequence of the blockage and the low velocity regions towards the hub 
revealed in this configuration, even at nominal conditions [31]. The flow cannot be guided accurately by the stator downstream, so the flow migrates to the $75 \%$ of the span. In particular, the low velocity of the flow in the relative frame results in an excessive under turning of the flow which leads to severe guidance losses and an extension of the blockage. The radial regions of lowvelocity represent the stator wake, which also interacts with the flow coming from the rotor. All these effects contribute to the establishment of a highly disturbed flow, with large areas of shear flow and high turbulence intensities. As a consequence, the number of ensemble averages required for convergence is notably enlarged, even though the acceptable criterion should be relaxed (to $2 \cdot 10^{-3}$ in this case). When the flow is reduced to a $70 \%$ of the nominal rate, the number of ensembles is increased as a consequence of the higher levels of turbulence, reaching up to 160180 in a wide number of locations within the inner section of the traverse. Tip zones are also significantly increased and calmed regions above the $75 \%$ of the span have now to be described with at least 40-50 ensembles.

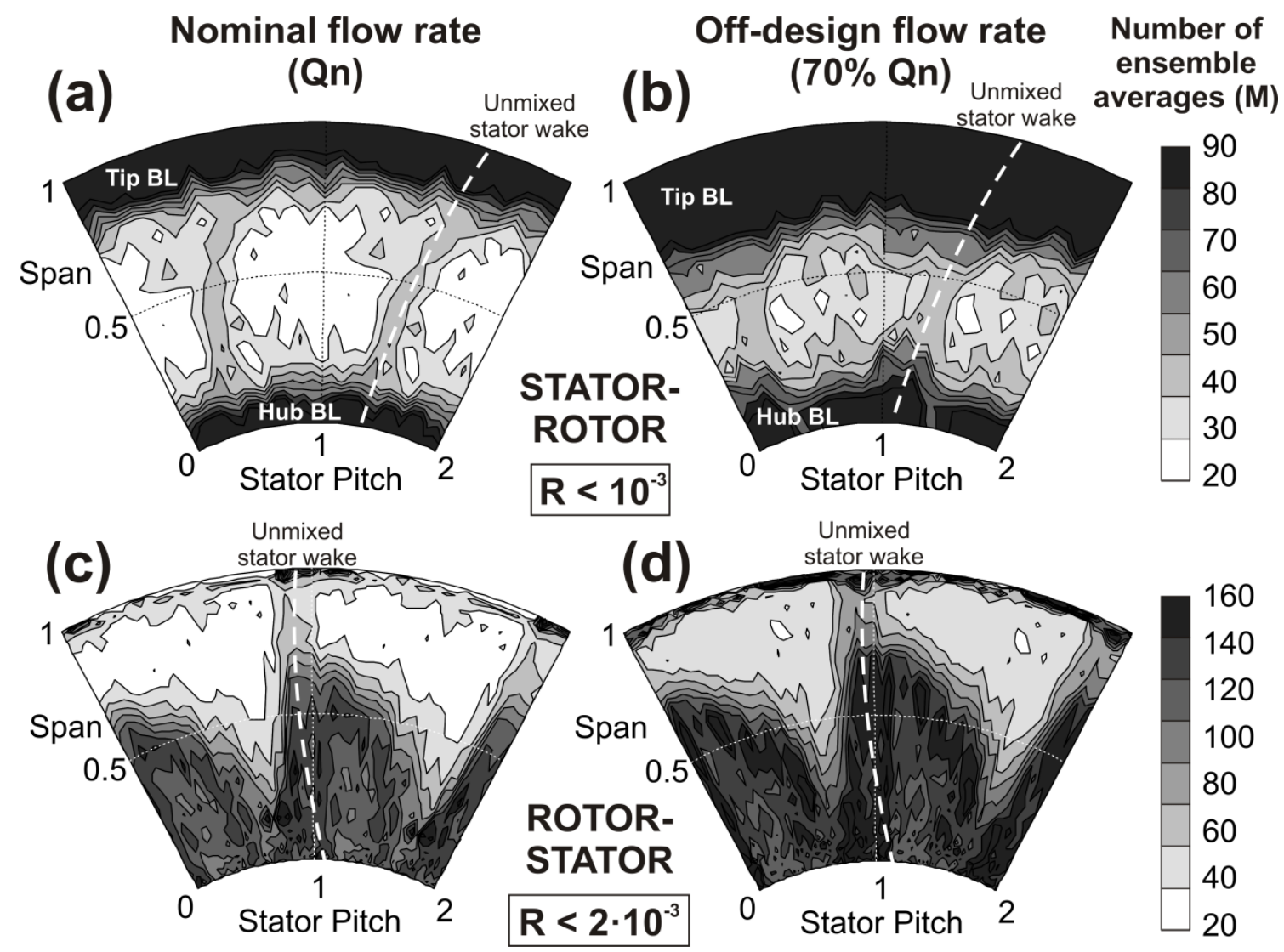

Figure 10. Maps of minimum ensemble averages required for the traverse measuring window to assure convergence at nominal and off-design conditions for S-R $(a, b)$ and R-S (c,d) configurations.

Correlation with turbulence data. We have derived in Annex $A$ that there is a clear correlation between the number of ensemble averages required for convergence and the time-averaged turbulence level of the flow. In particular, it is approximated that for typical convergence requirements $\left(10^{-3}\right)$, the number of ensembles to be adopted must be fixed in the range of one order of magnitude higher than the local averaged turbulence intensity expressed in percentage. For example, this means that for a typical turbulence level of $7.5 \%, 75$ ensemble averages should be fixed to assure convergence. This analytical estimation is validated presenting the timeaveraged maps of turbulence in the traverse window of the present database in figure 11. 
Comparison of internal regions with previous figure 10 reveals an absolute correlation between both sets of data. The flow structure is perfectly recovered with both representations, being the unmixed stator wake the most significant flow pattern. Not only there is a perfect correspondence between variable gradients, there is also a scaling ratio of order 10 between ensembles and turbulence intensities, as expected. In the case of S-R configuration, at nominal conditions, tip boundary layer exhibits turbulence levels up to $35 \%$ (here, the representation has been clipped to $25 \%$ in order to show wake turbulence, otherwise masked by maximum values in the endwall zones), indicating that approximately, 300-350 ensemble averages should have been employed during data acquisition for post-processing. At off-design conditions -(figure 11(b)-, maximum levels of turbulence in hub and tip regions are found to be around 20 and $50 \%$ respectively. This means that tip vortex (tangentially averaged in the time-averaging procedure) significantly increases the level of disorder in the flow, with respect to hub regions, advising the expectable requirement of 500 ensemble averages to capture correctly the unsteady scales of the tip vortex in the S-R configuration.

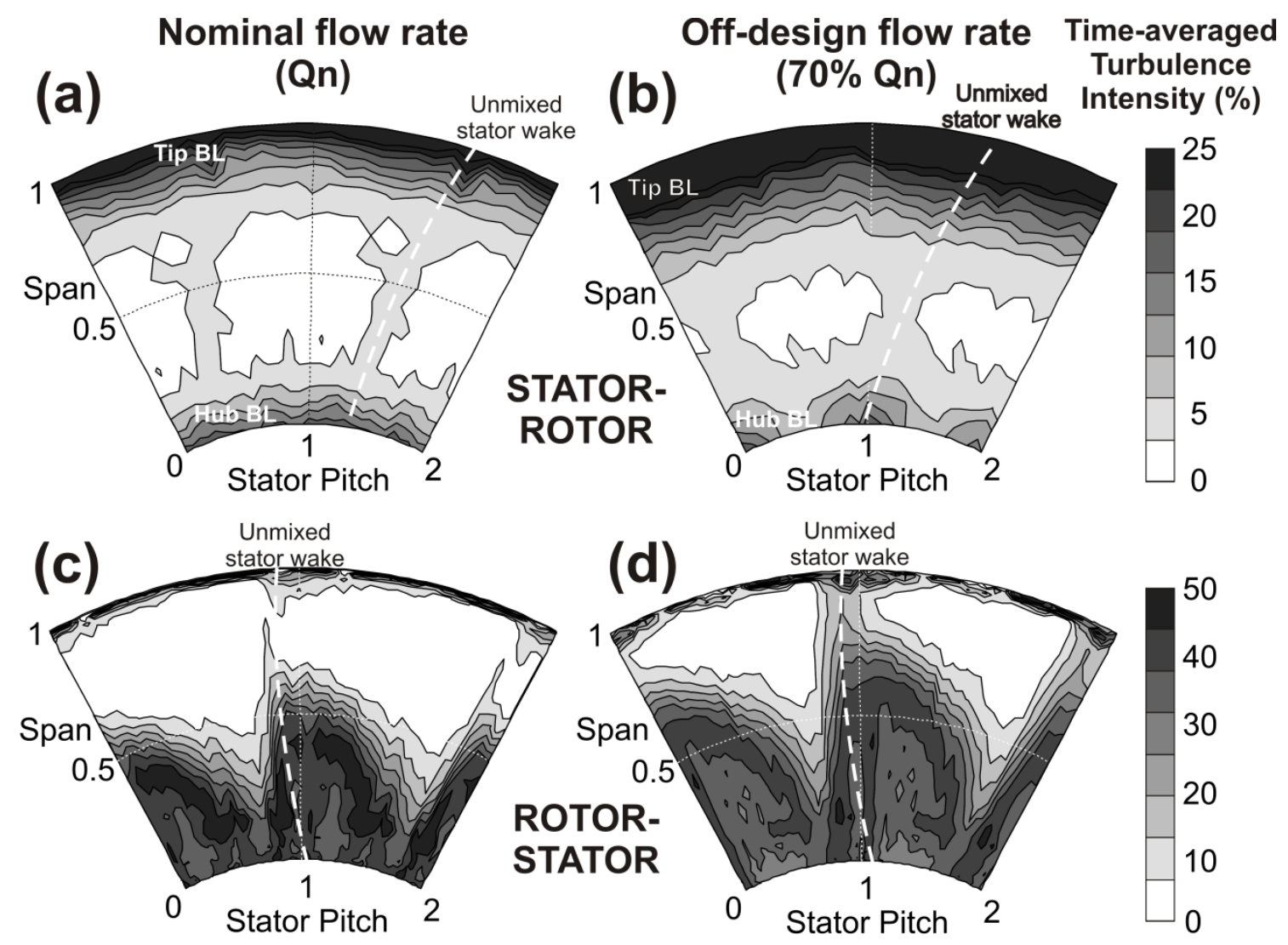

Figure 11. Map of time-averaged turbulence intensity for the traverse measuring window at nominal $(a, c)$ and off-design $(b, d)$ conditions for S-R and R-S configurations.

Additionally, maps of turbulence for the R-S configuration have been also represented in figure 11. It is interesting to note that maximum turbulence is found in the shear layers of the separated region in the hub. Moreover, in the core of the massive separation, the turbulent levels are lower because the velocity is also notably reduced and, as a consequence, the turbulent fluctuations are also not as high as in the instabilities of the shear layers. For both nominal and off-design conditions, these maximum turbulence levels reach up to $40-50 \%$, which implies an approximate number of 200-250 ensembles for a convergence criterion of $2 \cdot 10^{-3}$, in accurate concordance with previous results shown in figure 9 . Note that if a typical criterion of $10^{-3}$ is desired, the number of 
ensembles should be raised up to 500 , in a similar fashion to the expected values for the tip flow structures of the S-R configuration.

Precisely, because the election of the convergence threshold is of interest to characterize the level of convergence attained, a final analysis has been made correlating the maps of number of ensembles (for a particular residual level) with the turbulence distributions. In other words, maps shown in figures 10 and 11 have been cross-correlated to compare and quantify their similarities, varying the maps of ensembles required with limit residuals in the range of $10^{-1}$ to roughly $10^{-3}$. In the figure 12(a), the normalized correlation coefficient has been represented for the entire database (R-S and S-R configuration for both nominal and off-design conditions) as a function of the convergence threshold. Besides, the maximum number of ensembles required $\left(N_{\max }\right)$ in the most turbulent locations for every case has been also represented in the bottom plot of the figure. The results reveal that the zone of maximum correlation (higher than 0.95 ) is preserved when the convergence threshold is at least set to $10^{-2}$ (which represents roughly a $1 \%$ of discrepancy in the mean quadratic error during the ensembling procedure). It should be expected for the correlation coefficient to increase asymptotically towards unity, but it suddenly drops when the threshold is lower than $2 \cdot 10^{-3}$. Obviously, this an artificial effect caused by the limitation in the maximum number of ensembles possible for the experimental databases. This can be clearly noticed in the bottom plot when both S-R and R-S configurations reach their maximum number of available ensembles (100 and 200 respectively) at $2 \cdot 10^{-3}$ and afterwards the lines saturate for lower values of residual. Whatever the case, the idea to keep in mind is that notably high correlations are guaranteed if the convergence threshold is at least of 1 hundredth (the accurate interval is highlighted in grey in the plots).

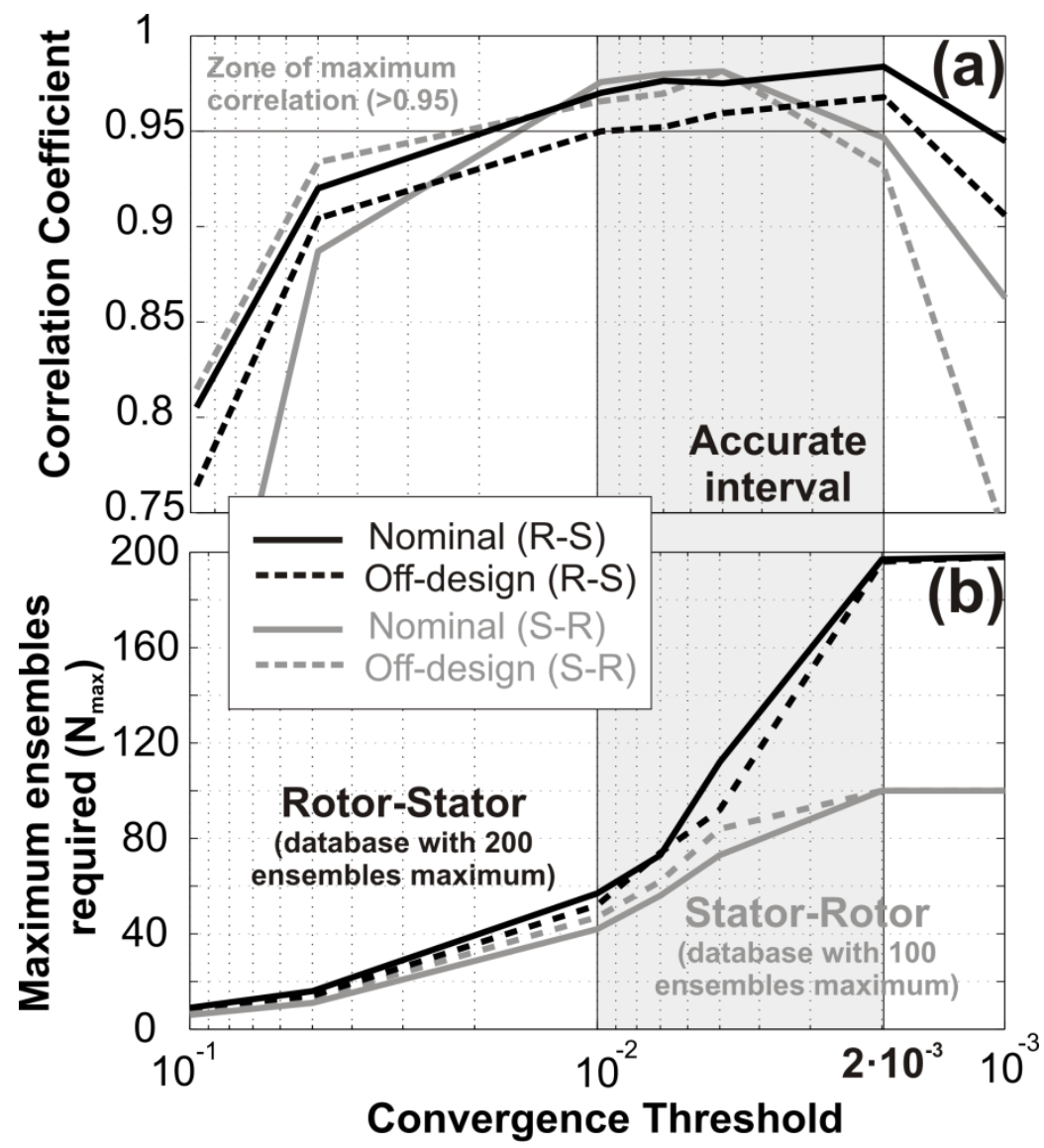

Figure 12. (a) Cross-correlation coefficientsbetween maps of ensemble averages and turbulence distributions as a function of different convergence thresholds for the entire database. (b) Maximum number of ensembles required. 
Impact of flow structures on number of samples. Now, the second indicator to assure converged statistics with respect to the number of samples per blade event is analyzed in this section. In particular, figure 13 presents the pitch-averaged distribution of the required number of samples for both S-R and R-S configurations with nominal (solid lines) and off-design conditions (dashed lines). This results have been obtained for a convergence criterion of $5 \cdot 10^{-3}$. Except for the contribution at endwall regions, the number of samples is quite moderate, with typical values ranging only from 30 to 50 . In the inner regions of the S-R configurations -figure $13(\mathrm{a})-$, only the periodic tangential displacement of the rotor wakes is sensed by the stationary probe, so a single (purely deterministic) periodic oscillation is perfectly described with few points, valid for the two flow rate conditions studied. At both hub and tip shear layers, the increment in the level of disorder of the flow (as well as additional oscillations and disturbances -instabilities- caused by shear flows) leads to the necessity of a larger number of samples (between 60 and 80 ).
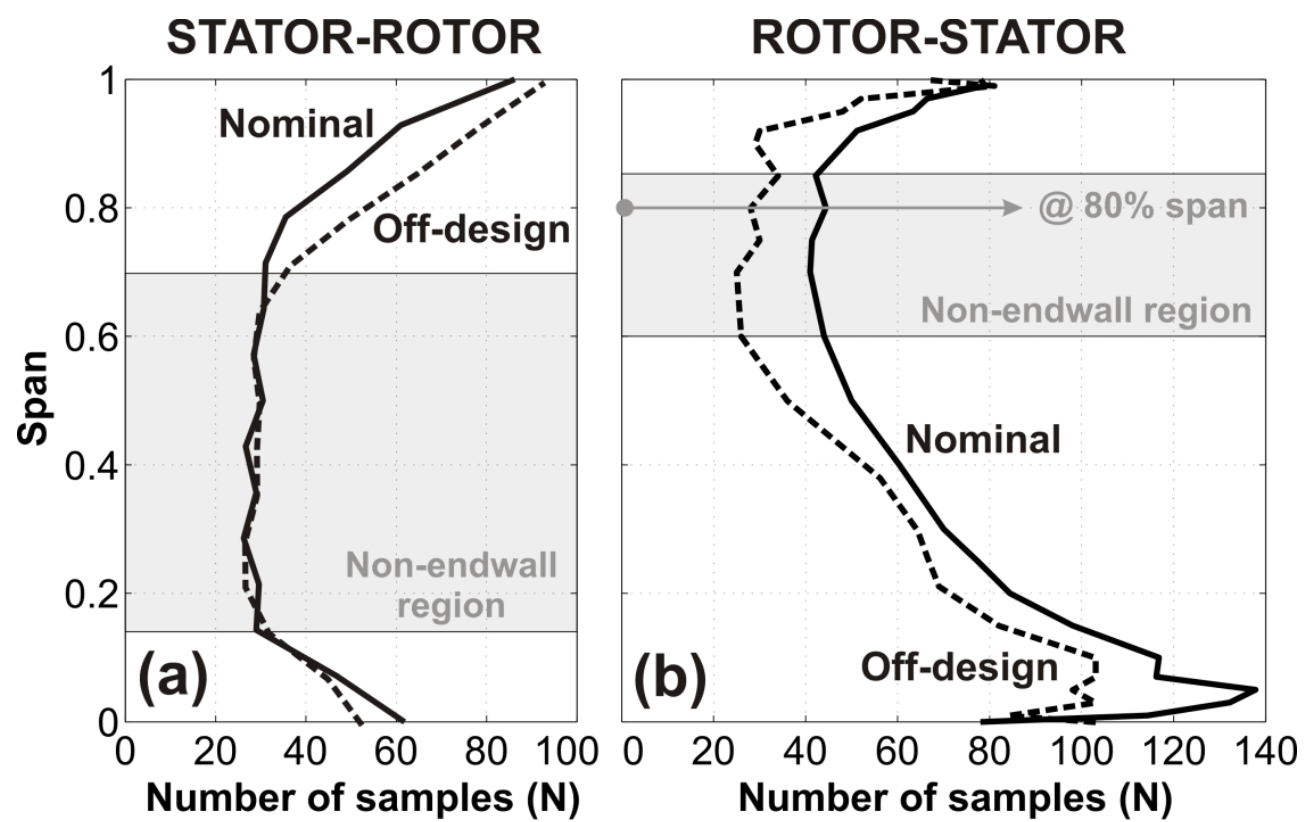

Figure 13. Pitch-averaged distribution of number of samples required for a minimum convergence threshold of $5 \cdot 10^{-3}$ for both S-R (a) and R-S (b) configurations stage downstream.

In the R-S configuration -figure 13(b)-, the large extension of the separated region in the hub zone (remember those turbulence maps in previous figure 11) provokes significant large values of required samples, only broken by a "calm window" within a 60 to an $85 \%$ of the span. Note that values in the order of a hundred of sampled points are needed in the inner half of the traverse, being even higher in the case of the nominal flow rate. All those locations out of the "non-endwall region", identified in the subplot by a grey horizontal band, are susceptible of vortical motions, high unsteadiness and three-dimensionality, with all the turbulence masking the (unmixed) rotor wake deficits. Only that internal region can be described as a planar flow, characterized by the periodic fluctuation of the rotor wakes passing by.

Consequently, figure 14 has been included to illustrate the nature of the rotor wakes at $80 \%$ of the midspan (within the region unaffected by the wall boundary layers) for the case of the R-S configuration at both nominal and off-design conditions. As expected, nominal wakes are thinner and more pronounced that off-design wakes, which are also more diffused and with higher 
thicknesses. These wakes have been represented here to compare them to the theoretical ones defined previously for figure 4 . If we take a look to the nominal trace, a wake width around 0.2 and a wake deficit also in the order of 0.2 can be reasonably adopted. With these values, the theoretical cosine wake predicts (see map on figure 4) around 29 samples to describe accurately the tangential gradients of the velocity traces. Analogous, the off-design trace presents a wake width of approximately 0.3 and a wake depth in the range of 0.15 , thus resulting in a theoretical requirement of 23 samples for convergence. It is evident that these real traces are more irregular than ideal wakes obtained from equation (7) -they have no plateau region outside the wake- so these estimations under predict a little bit the real needs, which are around 30 and 40 samples respectively in figure $13(\mathrm{~b})$. Here, the key point is that the theoretical wakes present extremely low levels of error in the estimation of the integral parameters of the wakes (below $0.5 \%$ in figure 5) when a residual criterion of $5 \cdot 10^{-3}$ is adopted. In the real practice, the error is unknown, but the number of ensembles required for that convergence level has been proven to be very similar, thus expecting that with such minimum thresholds, the tangential description of the wakes must be optimum (i.e., reduced discretization errors).

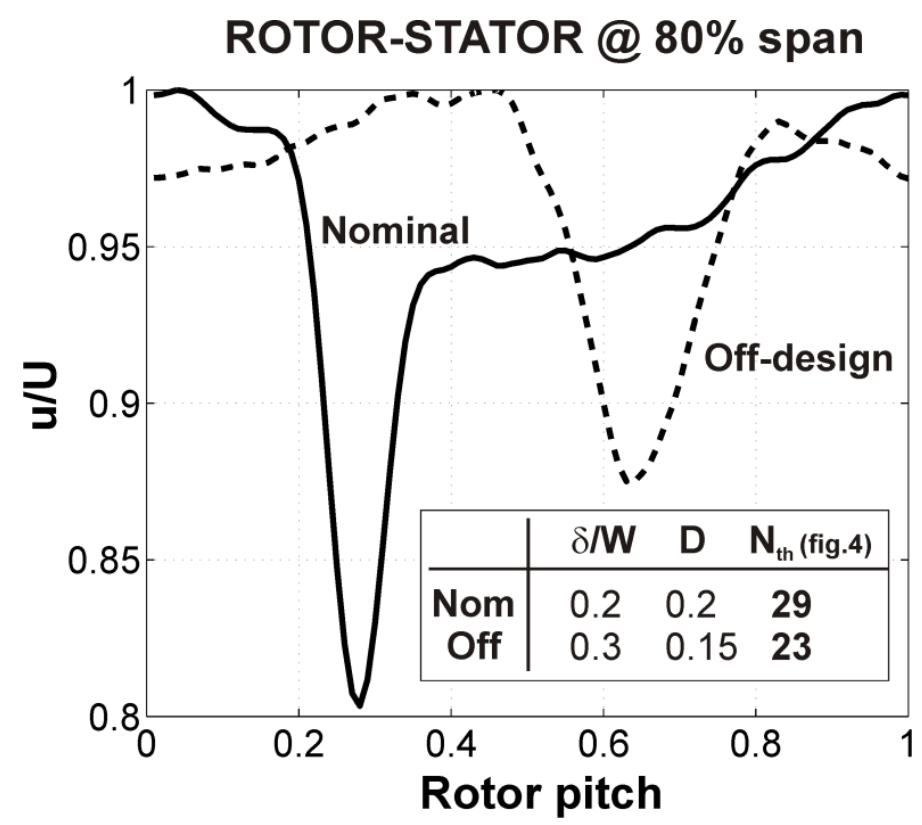

Figure 14. Representation of typical rotor wakes at $80 \%$ of the span in the R-S configuration. Theoretical number of samples required to assure convergence from wake parameters.

Outside of those regions characterized by a planar flow, it is clear that the irregularities and disorder associated to the turbulence of the flow play a significant role. Previous figure 13 revealed how the number of required samples is significantly increased when the turbulence intensity is enlarged close to the endwalls: all-scale fluctuations over the velocity traces contribute to complicate the tangential description of the traces. Therefore, it is quite intuitive that the level of turbulence must present a certain impact on the convergence for the number of samples.

Figure 15 shows two maps with the number of samples per blade event required to attain a convergence criterion of $5 \cdot 10^{-3}$ for the tangential description of the time-resolved velocity traces at every measuring point of the database. Both nominal and off-design results provide quite similar conclusions. Firstly, there is an evident link between this indicator and the overall level of turbulence, because those regions with large number of samples are coincident with the zones 
responsible for turbulence generation. In this case, 80-90 samples appear to be sufficient for the tangential description of the wakes. However, there is no direct correlation with the turbulence data (in a similar fashion to what happened before with the ensembles), as it is revealed in the annexed table embedded in the figure. The unmixed stator wakes, perfectly identifiable in figure 10, are now completely removed, which helps to reduce the total level of autocorrelation respect to the levels observed for the ensembling operator (figure 15(b) and left table). While the turbulence intensity in the endwall shear layers sets off large amount of unsteadiness and irregularities, the shear flow associated to unmixed stator wakes is not responsible for the generation of more disorder. It only reduces the local levels of streamwise velocity, enlarging the wake velocity deficit, and as a consequence, the maps of minimum samples are less correlated with the turbulent maps.

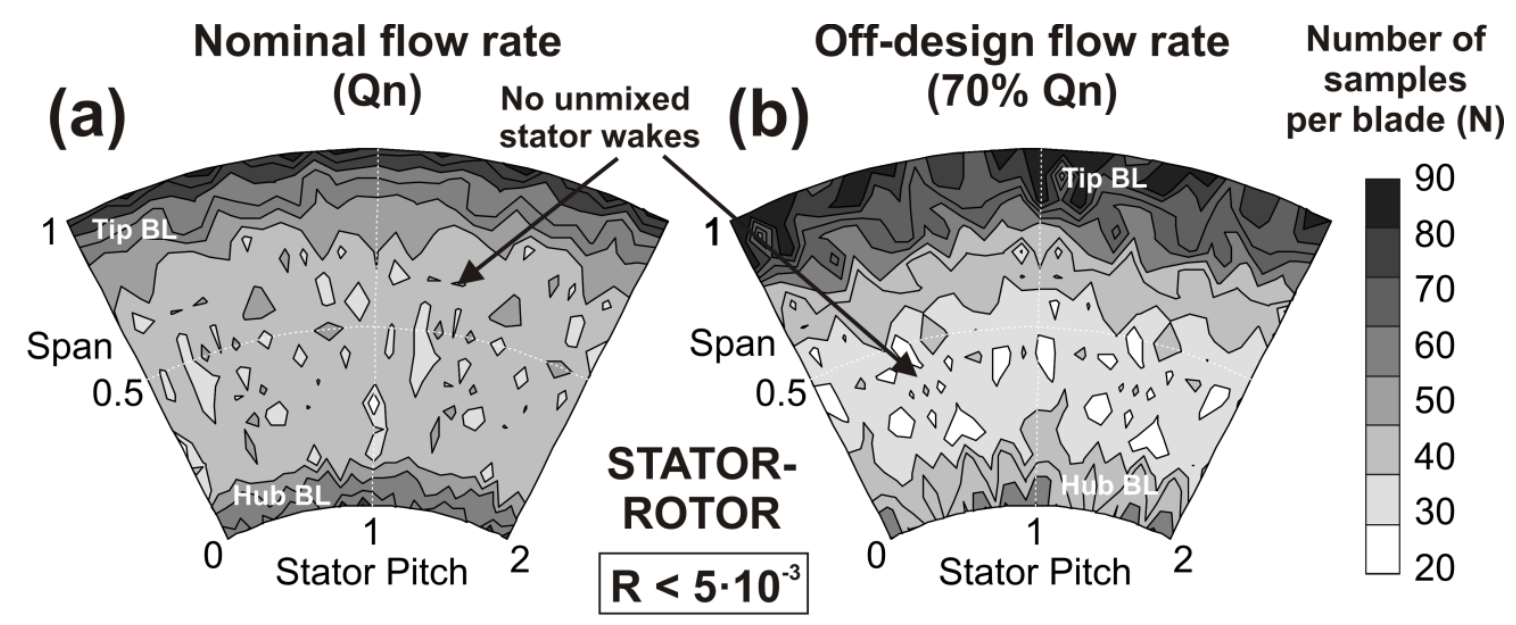

\begin{tabular}{ccc}
\multicolumn{3}{c}{$\begin{array}{c}\text { Correlation with Turbulence for } \\
\text { converged number of ensembles (M) }\end{array}$} \\
\cline { 2 - 3 } & $\mathbf{1 0}^{-2}$ & $\mathbf{5 \cdot 1 0}^{-3}$ \\
\hline Nom & 0.9757 & 0.9814 \\
Off & 0.9657 & 0.9817 \\
& (Very high correlation)
\end{tabular}

\begin{tabular}{cc}
\multicolumn{2}{c}{$\begin{array}{c}\text { Correlation with Turbulence for } \\
\text { converged number of samples }(\mathrm{N})\end{array}$} \\
\hline $\mathbf{1 0}^{-2}$ & $\mathbf{5 \cdot 1 0 ^ { - 3 }}$ \\
\hline 0.8132 & 0.8332 \\
0.7410 & 0.8264 \\
(Moderate correlation)
\end{tabular}

Figure 15. Maps of minimum number of samples required for the traverse measuring window to assure convergence (@ 5·10 $10^{-3}$ ) at nominal (a) and off-design (b) conditions for the S-R configuration.

\section{5.- CONCLUSIONS}

A technique to quantify the convergence of phase-locked averaging (PLA) processes when applied to velocity signals acquired with stationary probes in axial turbomachinery has been presented in a statistical framework. The study has also analyzed with the effect of number of periods (ensembles), resolution in which the averaged periods are reconstructed and turbulence intensity on the experimental accuracy of ensemble-averaged measurements in low-speed axial fans using fast-response probes. The procedure has been established in terms of convergence (residuals) between time-resolved traces retrieved using different sampling frequencies and number of total samples.

A concise review of the open literature has provided typical ranges for acquisition parameters used for axial fans and compressors when dealing with PLA techniques. In particular, the number 
of samples employed for ensemble-averaging the raw velocity traces, as well as the tangential discretization expressed as the number of sampling points per degree were shown to be essential selections to obtain an accurate statistical reduction of the experimental database. The great disparity in the range of these parameters is clearly related to the requirements of the measurements, pending on the variables to be analyzed, the local flow patterns or the global disorder of the flow as a consequence of the operating conditions.

Concerning the time-averaging operator, i.e, the number of samples $N$, it has been confirmed that the angular discretization must assure an overall residual in the order of $5 \cdot 10^{-3}$ to provide an accurate level of convergence respect to the integral parameters for the rotor wakes. The spatial distribution of samples required for a convergence threshold in the traverse measuring windows is not fully recovering the flow patterns (turbulent structures), and only a spanwise evolution shows a higher correlation with the instabilities and disorders associated to the endwall boundary layers.

On the other hand, it has been demonstrated a direct relationship between the number of ensemble averages and the turbulence levels of the flow in order to guarantee a certain level of convergence. An analytical expression for the estimation of the required ensembles as a function of local mean turbulence has been derived and contrasted experimentally. In essence, the selection of ensembles must be in the range of one order of magnitude higher than the turbulence level expressed in hundred percent if a typical $10^{-3}$ convergence criterion is adopted. However, it has been demonstrated that convergence thresholds in the order of $5 \cdot 10^{-3}$ and even $10^{-2}$ can be sufficient to provide correlations with the turbulent structures up to 0.95 . The influences of the operating conditions and the spanwise location of the measurements have been also explored, revealing that endwall zones and off-design conditions are critical contributors to increase the number of ensembles. Moreover, the tangentially-averaged impact of tip vortex structures in S-R configurations or the appearance of massive blocked regions near the hub in the R-S configuration were found to be complex three-dimensional mechanisms that would require up to 500 ensembles for a statistical convergence, thus doubling the characteristic values found in the shear flows linked to both rotor/stator wakes.

\section{ACKNOWLEDGEMENTS}

The authors acknowledge the support from the Research Project, "Aeroacoustic Characterization of Elliptic Airfoils in Turbulent Flow", ref. DPI-2009-13613, sponsored by the Spanish Ministry of Science and Innovation (MICINN).

\section{NOMENCLATURE}

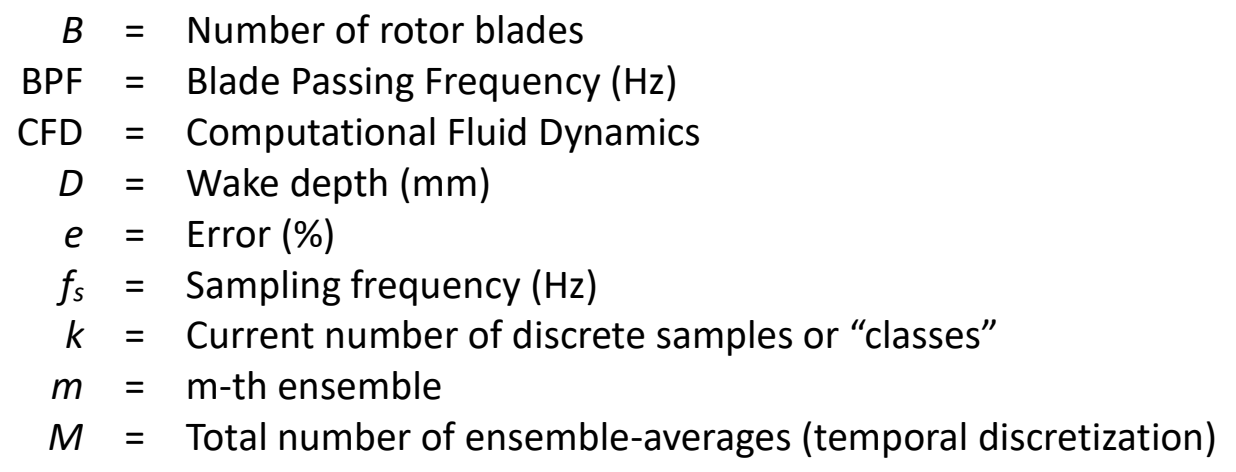




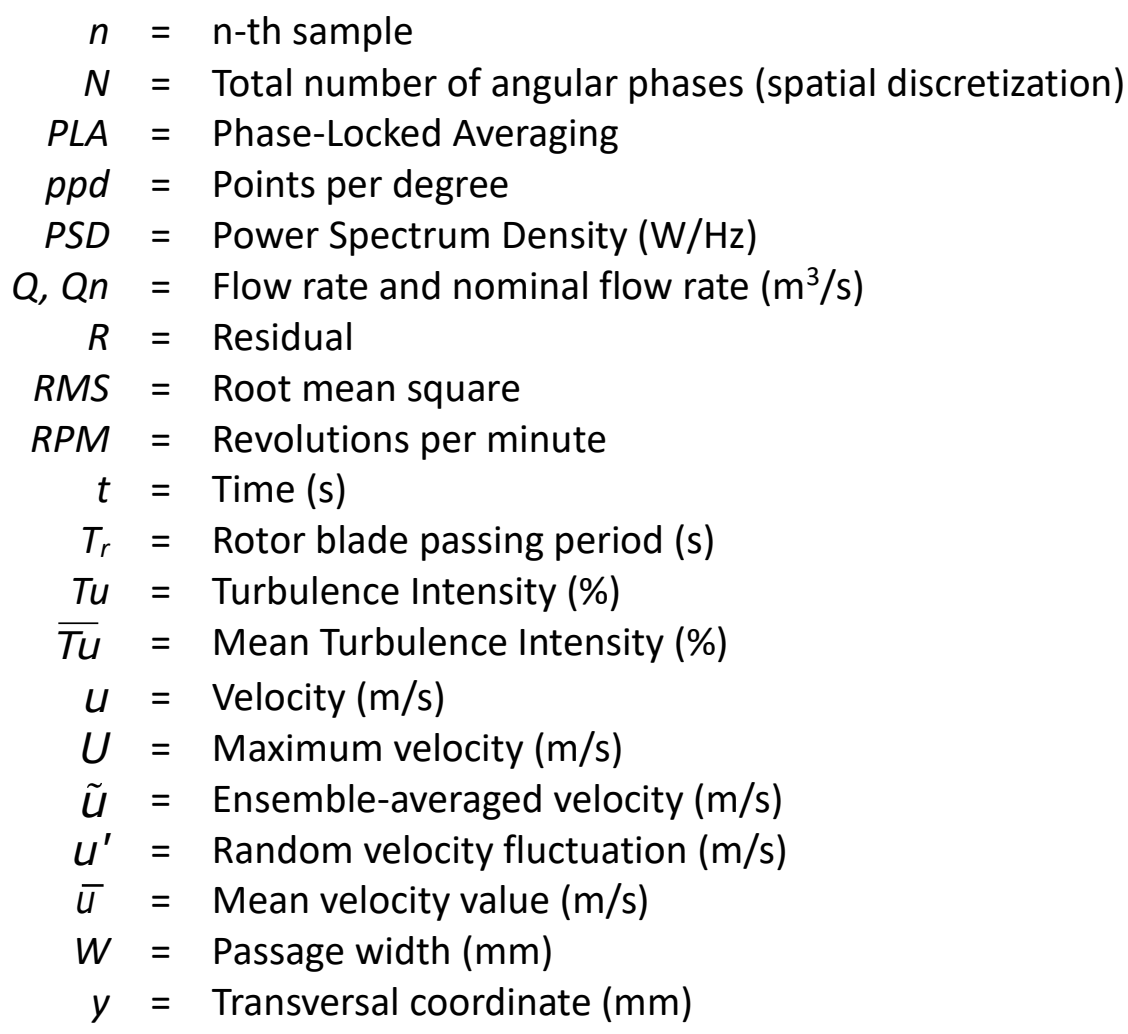

\section{Greek symbols}

$\delta=$ Wake width $(\mathrm{mm})$

$\delta^{*}, \delta_{\mathrm{p}}^{*}=$ Displacement thickness $(\mathrm{mm})$

\section{Superscripts and subscripts}

$$
\begin{aligned}
\sim & =\text { Ensemble-averaging operator } \\
- & =\text { Time-averaging operator } \\
m & =\text { Number of ensemble-average } \\
n & =\text { Number of angular phase } \\
t h & =\text { Theoretical } \\
\max & =\text { Maximum }
\end{aligned}
$$

\section{REFERENCES}

[1] Huyer, S.A., Snarski, S.R., 2003, "Analysis of a turbulent propeller inflow". ASME Journal of Fluids Engineering, 125, pp. 533-542.

[2] Kergourlay, G., Kouidri, S., Rankin, G.W., Rey, R., 2006, "Experimental investigation of the 3D unsteady flow field downstream of axial fans". Flow Measurement and Instrumentation, 17, pp. 303-314.

[3] Read, S., Elder, R.L., 1999, "The nature of wakes in multistage axial flow compressors". Proceedings of the IMechE - part A, Journal of Power and Energy, 213, pp. 275-289. 
[4] Mailach, R., Vogeler, K., 2004, "Aerodynamic blade row interactions in an axial compressor Part I: unsteady boundary layer development". ASME Journal of Turbomachinery, 126, pp. 3544.

[5] Velarde-Suárez, S., Ballesteros-Tajadura, R., Santolaria-Morros, C., Blanco-Marigorta, E., 2002, "Total unsteadiness downstream of an axial flow fan with variable pitch blades". ASME Journal of Fluids Engineering, 124, pp. 280-283.

[6] Prato, J., Lakshminarayana, B., Suryavamshi, N., 2001, "Three-dimensional flow field downstream of an embedded stator in a multistage axial flow compressor - Part I: steady and unsteady flow fields". Proceedings of the IMechE - part A, Journal of Power and Energy, 215, pp. 281-299.

[7] Lepicovsky, J., 2008, "Effects of a rotating aerodynamic probe on the flow field of a compressor rotor". Proceedings of the XIX Biannual Symposium on Measuring Techniques in Transonic and Supersonic Flow in Cascades and Turbomachines". Rhode-St-Génese, Belgium.

[8] Brunn, H.H., "Hot-Wire Anemometry Principles and Signal Analysis", 1995, Oxford University Press.

[9] Henderson, A.D., Walker, G.J., Hughes, J.D., 2006, "The influence of turbulence on wake dispersion and blade row interaction in an axial compressor". ASME Journal of Turbomachinery, 128, pp. 150-157.

[10] Senkter, A., Reiss, W., 1998, "Measurement of unsteady flow and turbulence in a low speed axial compressor", Experimental Thermal and Fluid Science, 17, pp. 124-131.

[11] Lakshminarayana, B., Poncet, A., 1974, "A method of measuring three-dimensional wakes in turbomachinery". ASME Journal of Fluids Engineering, 96 (2), pp. 87-91.

[12] Lakshminarayana, B., 1981, "Analytical and experimental study of flow phenomena in noncavitating rocket pump inducers". NASA Technical Report CR-3471.

[13] Camp, T.R., Shin, H.-W., 1995, "Turbulence intensity and length scale measurements in multistage compressors". ASME Journal of Turbomachinery, 117, pp. 38-46.

[14] Uzol, O., Camci, C., 2001, "The effect of sample size, turbulence intensity and the velocity field on the experimental accuracy of ensemble averaged PIV measurements", 4th International Symposium on Particle Image Velocimetry PIV'01 Paper 1096 Göttingen, Germany, September 17-19.

[15] Perrin, R., Cid, E., Cazin, S., Sevrain, A., Braza, M., Moradei, F., and Harran, G., 2007, “Phase averaged measurements of the turbulence properties in the near wake of a circular cylinder at high Reynolds number by 2C-PIV and 3C-PIV". Experiments in Fluids, 42, pp. 93-109.

[16] Ullum, U., Schmidt, J.J., Larsen, P.S., McCluskey, D.R., 1997, "Temporal evolution of the perturbed and unperturbed flow behind a fence: PIV analysis and comparison with LDA data", Proceedings of the 7th International Conference on Laser anemometry and applications, Karlsruhe, Germany, pp. 809-816.

[17] Wernert, P., Favier, D., 1999. "Considerations about the phase-averaging method with application to ELDV and PIV measurements over pitching airfoils". Experiments in Fluids, 27, pp. 473-483.

[18] Cavazzini G., Pavesi G., Ardizzon G., 2010, "Validation procedure of turbulent unsteady flows in a PIV analysis in the turbomachinery field", Proceedings of the IMechE - part A, Journal of Power and Energy, 224 (A5), pp. 679-689. 
[19] Clark, J.P., Grover, E.A., 2006, "Assessing convergence in predictions of periodic-unsteady flow fields". Proceedings of the ASME Turbo Expo 2006, Paper GT2006-90735, Barcelona, Spain.

[20] Tropea, C., Yarin, A.L., Foss, J.F. (editors), “Handbook of Experimental Fluid Mechanics”, 2007, Springer.

[21] Goto, A., 1992, "Three-dimensional flow and mixing in an axial flow compressor with different rotor tip clearences", ASME Journal of Turbomachinery, 114, pp. 675-685.

[22] Fernández Oro, J.M., Blanco Marigorta, E., Argüelles Díaz, K.M., Ballesteros-Tajadura, R., 2009, "Forced and unforced unsteadiness in an axial turbomachine", Experimental Thermal and Fluid Science, 33 (3), pp. 449-459.

[23] Cavazzini, G., Dazin, A., Pavesi, G., Dupont, P., Bois, G., 2012, "Post-processing methods of PIV instantaneous flow fields for unsteady flows in turbomachines" -chap.4 in "The particle image velocimetry - Characteristics, limits and possible applications". PhD. Giovanna Cavazzini (Ed.), ISBN: 978-953-51-0625-8, InTech.

[24] Heinz, O., Ilyushin, B., Markovic, D., 2004, "Application of a PDF method for the statistical processing of experimental data". International Journal of Heat and Fluid Flow, 25, pp. 864874.

[25] Hill, P.G., Schaub, U.W., Senoo, Y., 1963, "Turbulent wakes in pressure gradients", ASME Journal of Applied Mechanics, 30 (4), pp. 518-524.

[26] Van Zante, D.E., Adamczyk, J.J., Strasizar, A.J., Okiishi, T.H., 2002, "Wake recovery performance benefit in a high-speed axial compressor", ASME Journal of Turbomachinery, 124, pp. 275-284.

[27] Greitzer, E.M., Tan, C.S., Graf, M.B., "Internal Flow. Concepts and Applications", 2004, Cambridge University Press.

[28] Fernández Oro, J.M., Argüelles Díaz, K.M., Santolaria Morros, C., Blanco Marigorta, E., 2007, "Unsteady flow and wake transport in a low-speed axial fan with inlet guide vanes", ASME Journal of Fluids Engineering, 129, pp. 1015-1029.

[29] Fernández Oro, J.M., Argüelles Díaz, K.M., Santolaria Morros, C., Blanco Marigorta, E., 2007, "On the structure of turbulence in a low-speed axial fan with inlet guide vanes", Experimental Thermal and Fluid Science, 32 (8), pp. 316-331.

[30] Uzol, O., Brzozowski, D., Chow, Y.-C., Katz, J., Meneveau, C., 2007, "A database of PIV measurements within a turbomachinery stage and sample comparisons with unsteady RANS", Journal of Turbulence, 8, N10.

[31] Galdo Vega, M., Fernández Oro, J.M., Santolaria Morros, C., Argüelles Díaz, K.M., "Effect of rotor-stator configuration in the generation of vortical scales and wake mixing in single stage axial fans. Part I: LES modelling and experimental validation", Proceedings of the ASME FEDSM 2013, Paper FEDSM2013-16432, Incline Village, USA.

[32] Inoue, M., Kuroumaru, M., 1989, "Structure of tip clearance flow in an isolated axial compressor rotor", ASME Journal of Turbomachinery, 111, pp. 250-256.

[33] Dong, Y., Cumpsty, N.A., 1990, "Compressor blade boundary layers: Part 2 - Measurements with incident wakes", ASME Journal of Turbomachinery, 112, pp. 231-241.

[34] Witkowski, A.S., Chmielniak, T.J., Strozik, M.D., 1996, "Experimental study of a 3D wake decay and secondary flows behind a rotor blade row of a low speed axial compressor", Proceedings of the ASME Turbo Expo 1996, ASME Paper 96-GT-415, Birmingham, UK. 
[35] Halstead, D.E., Wisler, D.C., Okiishi, T.H., Walker, G.J., Hodson, H.P., Shin, H.W., 1997, "Boundary layer development in axial compressors and turbines: Part 1 of 4 - Composite picture”, ASME Journal of Turbomachinery, 119, pp. 114-127.

[36] Furukawa, M., Saiki, K., Nagayoshi, K., Kuroumaru, M., Inoue, M., 1998, "Effects of stream surface inclination on tip leakage flow fields in compressor rotors", ASME Journal of Turbomachinery, 120, pp. 683-692.

[37] Quinlan, D.A., Bent, P.H., 1998, "High frequency noise generation in small axial fans", Journal of Sound and Vibration, 218 (2), pp. 177-204.

[38] Shiomi, N., Cai, W.X., Muraoka, A., Kaneko, K., Setoguchi, T, 2003, "Internal flow of high specific-speed diagonal-flow fan (rotor outlet flow fields with rotating stall)", International Journal of Rotating Machinery, 9, pp. 337-343.

[39] Jang, C.M., Sato, D., Fukano, T., 2005, “Experimental analysis on tip leakage and wake flow in an axial flow fan according to flow rate", ASME Journal of Fluids Engineering, 127, pp. 322329.

[40] Henner, M., Moreau, S., Neal, D., Labordiere, J., 2007, "Validation of 3D rotor-stator URANS in automotive engine cooling fan systems", Proceedings of the 8th International Symposium on Experimental and Computational Aerothermodynamics of Internal Flows, ISAIF08-0072.

[41] Vad, J., Kawedikha, A.R.A., Horváth, C.S., Balczó, M., Lohász, M.M., Régert, T., 2007, "Aerodynamics effects of forward blade skew in axial flow rotors of controlled vortex design", Proceedings of the IMechE - part A, Journal of Power and Energy, 221, pp. 1011-1023.

[42] Eberlinc, M., Sirok, B., Dular, M. et al., 2011, "Modification of axial fan flow by trailing edge self-induced blowing", ASME Journal of Fluids Engineering, 131 (11), No. 111104.

[43] Shiomi, N., Kinoue, Y., Jin Ying, Z. et al., 2009, "Flow fields with vortex in a small semi-open axial fan", Journal of Thermal Science, 18 (4), pp. 294-300.

[44] Hurault, J., Kouidri, S., Bakir, F. et al., 2010, "Experimental and numerical study of the sweep effect on three-dimensional flow downstream of axial flow fans", Flow Measurement and Instrumentation, 21 (2), pp. 155-165.

[45] Sarraf C., Nouri, H., Ravelet, F., et al., 2011, "Experimental study of blade thickness effects on the overall and local performances of a controlled vortex designed axial-flow fan", Experimental Thermal and Fluid Science, 35 (4), pp. 684-693.

[46] Eberlinc, M., Sirok, B., Hocevar, M., 2009, "Experimental investigation of the interaction of two flows on the axial fan hollow blades by flow visualization and hot-wire anemometry". Experimental Thermal and Fluid Science, 33 (5), pp. 929-937.

\section{LIST OF FIGURES}

Fig. 1. Phase-locked averaging technique.

Fig. 2. Convergence (a) and tangential accurary (b) for time-resolved traces.

Fig. 3. Convergence histories for ensembling (a) and sampling (b) operators.

Fig. 4. Influence of the wake profile in the number of sample points required for a converged tangential accuracy. (a) Minimum number of samples. (b) Wake profile. 
Fig. 5. Estimation of the error in the wake displacement thickness for a wake profile discretized with $\mathrm{N}$ points given a minimum residual criterion of $5 \cdot 10^{-3}$.

Fig. 6. Measuring windows (a) and spatial discretizations (b).

Fig. 7. Residual histories for velocity and flow angle, stage downstream of the R-S configuration. Influence of the operating conditions at midspan (a) and boundaries (b) for the ensemble convergence.

Fig. 8. Residual histories for velocity and flow angle, rotor downstream of the S-R configuration. Influence of the operating conditions at midspan (a) and boundaries (b) for the samples convergence.

Fig. 9. Power spectrum density for instantaneous velocity signals, rotor downstream of the S-R configuration (a) and at stage downstream (b) for midspan and boundary locations.

Fig. 10. Maps of minimum ensemble averages required for the traverse measuring window to assure convergence at nominal and off-design conditions for $S-R(a, b)$ and R-S $(c, d)$ configurations.

Fig. 11. Map of time-averaged turbulence intensity for the traverse measuring window at nominal $(a, c)$ and off-design $(b, d)$ conditions for S-R and R-S configurations.

Fig. 12. (a) Cross-correlation coefficients between maps of ensemble averages and turbulence distributions as a function of different convergence thresholds for the entire database. (b) Maximum number of ensembles required.

Fig. 13. Pitch-averaged distribution of number of samples required for a minimum convergence threshold of $5 \cdot 10^{-3}$ for both S-R (a) and R-S (b) configurations stage downstream.

Fig. 14. Representation of typical rotor wakes at $80 \%$ of the span in the R-S configuration. Theoretical number of samples required to assure convergence from wake parameters.

Fig. 15. Maps of minimum number of samples required for the traverse measuring window to assure convergence (@ 5.10 $10^{-3}$ ) at nominal (a) and off-design (b) conditions for the S-R configuration.

\section{HIGHLIGHTS}

\#1. Technique to quantify the convergence of phase-lock averaging in axial flow fans.

\#2. Influence of required number of ensembles and total number of samples is addressed.

\#3. Number of required ensembles depends directly on the time-resolved turbulence level.

\#4. Number of samples for the averaged periods depends on the wake integral parameters.

\#5. Influence of the operating conditions and the spanwise locations is also explored. 


\section{ANNEX A \\ ESTIMATION OF MINIMUM NUMBER OF ENSEMBLE-AVERAGES TO ASSURE CONVERGENCE}

The expansion of equation (1), keeping in mind the ensemble-averaging process illustrated in figure 1 for $M$ realizations, can be expressed as:

$$
e_{n}^{M)}=\frac{u_{n}^{(1)}+u_{n}^{(2)}+\ldots+u_{n}^{(M)}}{M}
$$

where superscripts (1), (2),.., (M) stand for every one of the $m$-th ensembles available in the experimental measurements and $n$ represents every phase per blade event. Similarly, if we consider now $M-1$ ensembles, it gives:

$$
\operatorname{eif}_{n}^{M-1)}=\frac{u_{n}^{(1)}+u_{n}^{(2)}+\ldots+u_{n}^{(M-1)}}{M-1}
$$

Subtracting equation A.2 from equation A.1, it is possible to obtain the following relationship for the difference between $(M)$ and $(M-1)$ ensembles included in equation (5):

$$
Q i \delta_{n}^{M)}-U \delta_{n}^{M-1)}=\frac{-u_{n}^{(1)}-u_{n}^{(2)}-\ldots-u_{n}^{(M-1)}-u_{n}^{(M)}+M u_{n}^{(M)}}{M(M-1)}
$$

After some algebra, the difference is reformulated just in terms of the ensemble-averaged value with $M$ realizations, yielding:

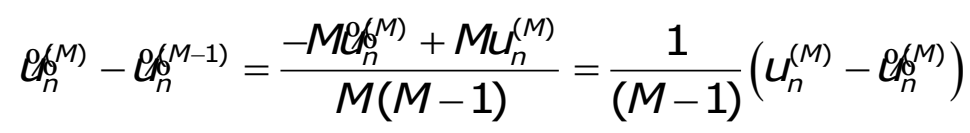

Now, reordering the residual definition in equation (5), we obtain:

$$
\left.\frac{R^{2}}{N}\left[\sum_{n=1}^{N} \operatorname{eg}_{n}^{M}\right]^{2}\right]^{2}=\sum_{n=1}^{N}\left(e_{n}^{M)}-e_{n}^{M-1)}\right)^{2}
$$

Considering that when $M$ is large enough, convergence is fully satisfied so increasing the number of ensemble averages results in no further improvements of the statistical representation, we can introduce the time averaging, giving: $\sum_{n=1}^{N} \mathcal{C} \sigma_{n}^{M)} \cong N \bar{u}$. Now, substituting this expression in (A.5): 


$$
R^{2} \bar{u}^{2} N=\frac{1}{(M-1)^{2}} \sum_{n=1}^{N}\left(u_{n}^{(M)}-Q i_{n}^{M)}\right)^{2}
$$

On the other hand, taking back to the definition of the unsteady ensemble-averaged turbulence in equation (3), we can reorder to express:

$$
\left[T u_{n}^{(M)} e f_{n}^{(M)}\right]^{2} M=\sum_{m=1}^{M}\left(u_{n}^{(m)}-Q \theta_{n}^{(M)}\right)^{2}
$$

Note that we have a quadratic difference $\left(u_{n}^{()}-\theta 0_{n}^{(M)}\right)^{2}$ in both summations in equations (A.6) and (A.7). However, in the first one it must be evaluated for all the phase samples, while in the second is for all the ensemble samples. To overcome this problem, we define the double summation for all the phases and all the ensembles which can be easily split into two terms if the $M$-th ensemble is isolated:

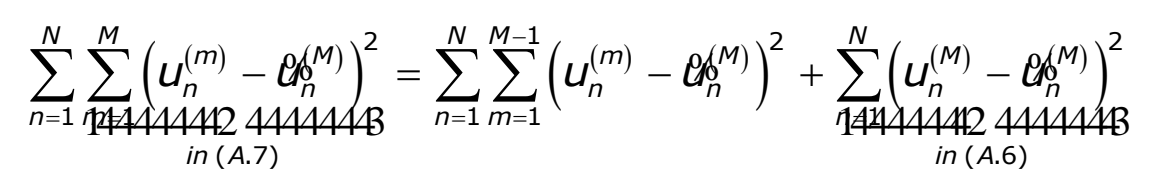

Note that in this expression, the summations involved in equations (A.6) and (A.7) are recovered. After substituting, it is direct to obtain the following relationship:

$$
\sum_{n=1}^{N}\left[T u_{n}^{(M)} e d_{n}^{(M)}\right]^{2} M=\sum_{n=1}^{N} \sum_{m=1}^{M-1}\left(u_{n}^{(m)}-e d_{n}^{(M)}\right)^{2}+(M-1)^{2} R^{2} \bar{u}^{2} N
$$

Obviously, if all this derivation is repeated from the beginning, but established between $(M+1)$ and (M) ensembles, we would obtain the same equation (A.9) updated for $M+1$ ensembles. In other words:

$$
(M+1) \sum_{n=1}^{N}\left[T u_{n}^{(M+1)} e 8_{n}^{(M+1)}\right]^{2}=\sum_{n=1}^{N} \sum_{m=1}^{M}\left(u_{n}^{(m)}-e d_{n}^{(M+1)}\right)^{2}+M^{2} R^{2} \bar{u}^{2} N
$$

Revisiting the definition of the unsteady definition in equation (3), we can express the timeresolved turbulence for $(M)$ ensembles as:

$$
T u_{n}^{(M)}=\frac{1}{\left.G_{n}^{M}\right)} \sqrt{\frac{1}{M} \sum_{m=1}^{M}\left(u_{n}^{(m)}-Q_{n}^{(M)}\right)^{2}}
$$

Once again, taking advantage of the consideration that convergence has been met, it is realizable to consider that $T u_{n}^{(M+1)} \cong T u_{n}^{(M)}$ and $C\left(\delta_{n}^{(M+1)} \cong e\left(\delta_{n}^{M)}\right.\right.$, so the double summation in (A.10) can be easily replaced with the unsteady turbulence in (A.11):

$$
(M+1) \sum_{n=1}^{N}\left[T u_{n}^{(M+1)} e_{n}^{(M+1)}\right]^{2}=\sum_{n=1}^{N} M\left(T u_{n}^{(M)} e_{n}^{(M)}\right)^{2}+M^{2} R^{2} \bar{u}^{2} N
$$


And finally give:

$$
(M+1) \sum_{n=1}^{N}\left(\theta \theta_{n}^{(M+1)} T u_{n}^{(M+1)}\right)^{2}-M \sum_{n=1}^{N}\left(\theta \theta_{n}^{(M)} T u_{n}^{(M)}\right)^{2} \cong \sum_{n=1}^{N}\left(\theta \delta_{n}^{M)} T u_{n}^{(M)}\right)^{2}=R^{2} N M^{2} \bar{u}^{2}
$$

To conclude, considering again the definition for the phase-averaged turbulence, it is possible to derive a relationship between the required number of samples to obtain accurate results within a fixed convergence criterion and the local turbulence level:

$$
\sum_{n=1}^{N} \vec{d}_{n}^{(2)}=R^{2} N M^{2} \bar{u}^{2}
$$

Rearranging a little bit further, the ensemble-averaged turbulence intensity (unsteady) is then transformed into a characteristic mean value, by means of the time-averaging operator. In other words:

$$
\frac{1}{N} \sum_{n=1}^{N}{\overline{d_{n}^{2}}}^{(M)}=\overline{{\overline{d_{n}^{22}}}^{(M)}}=R^{2} M^{2} \bar{u}^{2}
$$

Left-hand side in eq. (A.15) computes the mean-time fluctuation level. Precisely, the ratio between this fluctuation and the mean-time velocity magnitude determines the turbulence kinetic energy:

$$
\frac{1}{N \bar{u}^{2}} \sum_{n=1}^{N} \vec{d}_{n}^{(2)}=\overline{T u}^{2}=R^{2} M^{2}
$$

As a consequence, we can definitively provide a representative order of magnitude for $M$, considering that $M$ turns to be only a function of the residual and the time-averaged turbulence of the traces:

$$
M \cong \frac{1}{R} \overline{T u}
$$


This document is a pre-print version of the scientific paper published by Elsevier. It has been released by the authors to fulfill all the publisher requirements established for Article Sharing: https://www.elsevier.com/about/policies/sharing

\section{(9) $\Theta \Theta \Theta$}

(C) 2019. This manuscript version is made available under the Creative Commons Attribution-NonCommercial-NoDerivatives 4.0 International License (CC-BY-NC-ND 4.0 license) http://creativecommons.org/licenses/by-nc-nd/4.0/ 